Nomenclatura y Población Indígenas de la Antigua Jurisdicción de
Cali a Mediados del Siglo XVI 


\section{INDICE DE ILUSTRACIONES}

TABLAS

Pág. No.

1. Otros nombres mencionados en documentos de la época, no registrados en los autos del censo de 1552

2. Pueblos montañeses, dados por cargueros (tamemes) en la visita de 1559

3. Cargas traídas por indios montañeses desde el puerto de la Buenaventura hasta Cali - año de 1551

4. Encomiendas de la jurisdicción de Cali, censadas en el año de 1552 .

5. Las encomiendas de la jurisdicción de Cali: Cuadro comparativo, 1552-1559 (exclusa "la provincia de la montaña", no censada en 1552).

464

\section{MAPAS}

1. El Departamento del Valle del Cauca, Colombia

467

2. Area del antiguo distrito de Cali, censada en $1552 \quad 468$

3. Mapa actual de territorio censado en 1552

469

\section{LAMINAS}

1. Paisaje de la Cordillera entre Ylama y Vijes

470

2. Nacimiento del Río Pavas, mirando hacia el valle de Lile

3. Valle del Dagua medio. En el fondo, la montaña de los Papagayeros

4. Cabeceras del Río Bitaco

5. Vallecito de la Cordillera, a unos kilómetros de Ocache

6. El alto Río Jamundí

7. El valle del Aguamono (Restrepo), visto desde el Norte

8. El valle del Río Grande y el del Aguamono, visto del Sur 
Los límites del antiguo distrito de Cali, tal como los fijó el marqués Don Francisco Pizarro en agosto de 1539, abarcaban -aunque de manera algo imprecisa- la mayoría de los términos que hoy componen el Departamento del Valle del Cauca (1). Igual qu otras de las regiones del Occidente de Colombia, ésta había sido sujeta a innumerables invasiones en el milenario proceso de migración y poblamiento en el continente suramericano, pues ofrecía rutas fáciles de comunicación y a la vez una tierra privilegiada para colonización. En el momento del descubrimiento español, estaba habitada por decenas de tribus y sub-tribus cuyos orígenes y filiaciones son todaví materia de especulación y de hipótesis diversas.

Una de las principales dificultades que han encontrado etnógrafos e historiadores para clasificar a estas gentes, ha sido la falta de material lingüístico. El idioma, o los idiomas, que hablaban los moradores autóctonos se extinguieron sin que nadie se interesara por apuntar siguiera las palabras básicas de ellos, y no pasan de veinte los nombres -topónimos, apellidos y gentilicios - que mencionan los cronistas de li conquista de esta región.

Por lo tanto, reviste particular interés un documento, hasta ahora inédito, que conserva en el Archivo General de Indias como anexo al voluminoso expediente de it residencia tomada al oidor Francisco Brizeño en 1553-1554(2).Es éste el acta de visita de los naturales encomendados a vecinos de cali, que se realizó entre julio y diciembre de 1552, por orden del mismo Brizeño(3). En esta primera visita, qu antecedió en siete años la de la tasación de tributo que hizo el licenciado Tomá López, se empadronaron a todos los indios útiles o de servicio en veinte de lat veintitrés encomiendas del distrito. El documento registra por sus apellidos a 3.344

(1) Con base en las no muy precisas informaciones de que disponía, Pizarro decretó que call tuviera jurisdicción desde el río Timba hasta el confín del distrito de Anserma, en la band hacia Bug". Empleados Públicos de Cauca: AGI: Justia:

AGI: Justicia: Legajo 575. El documento, que consta de 23 hojas (45 páginas) numeradas sin relación a la foliación de las otras piezas del expediente, es un a copia certificada hecha por

3) Brizeño Rodrigo Hernandez el 12 de mayo de 1554, de las actas de 1552.

Real de Sa la ortografía que él usaba), nombrado oidor de la nuevamente creada Audieneia Real de Santa Fe del Nuevo Reino de Granada, había llegado de España con el encargo de tomar la residencia del Adelantado Don Se bastián de Belalcázar, conquistador y primer tituta de la Gobernación de Popayán. Destituído y condenado Belalcázar, Brizeño quedó pa gobernador interino, prosiguiendo la residencia de los tenientes y capitanes de aquel, hasia 376 tributarios, pertenecientes a 62 cacicazgos diferentes. Refiere además, 35 nombres de lugares y de tribus, 19 de los cuales no se mencionan, que sepamos, en otro documento alguno.

Es' este empadronamiento de 1552 el motivo de los presentes apuntes. Lo presentamos en las páginas que siguen, arreglados los sesenta y dos roles en una lista maestra por orden alfabético, con la identificación del pueblo, cacique y zona geográfica correspondiente a cada apellido. A este nomenclador van agregadas unas Tablas que completan el elenco con unos otros nombres propios de la misma región, sacados de diferentes documentos de la época.

Excluyendo de las listas de 1552 las repeticiones (hay, por ejemplo, 21 hombres de nombre Atende, distribuídos por doce cacicazgos), los nombres españoles y los moquetes puestos por los encomenderos (por todos, 70), y "un yndio sin nombre", quedan 2.303 apellidos indígenas diferentes. Algunos de estos deben ser variantes - por fonética local o por descuido del intérprete o del escribiente - de un mismo nombre: v gr., Bos: Boz, y tal vez, Guinxe: Guinçe. Sin embargo, pensando en las semejanzas engañosas que suelen presentar vocabularios y nomenclaturas en todo idioma, es evidente que los parecidos pueden ser despistadores. Dos nombres son foráneos: Nicaragua y Guancavelico, que más que apellidos son apodos, y sin duda hay otros que son extraños al distrito, aunque por lo general los yanaconas no eran agregados a las tribus locales, y estaba prohibido llevar indios de servicio "fuera de su natural".

$\mathrm{Si}$ a los 2.338 nombres diferentes del empadronamiento de 1552 , agregamos los 40 que se pueden sacar de otros documentos anteriores a 1575 como propios de la región del primer censo, tenemos un total de 2.378 nombres, sin contar "los montañeses" registrados en la tasación que se hizo en 1559 , que más tarde anotamos, y uno dudoso que suministra Andagoya.

El área comprendida en la visita de 1552 , era de unos 3.000 kilómetros cuadrados. (Véase el mapa, No. 2). En términos geográficos, abarcaba la banda izquierda del río Cauca y las vertientes a ésta desde el río Timba hasta un punto no precisado que puede haber sido el Mediacanoa (originalmente, Meacanoa), y al Oeste del divisorio de las aguas de la Cordillera Occidental, a toda la cuenca del Dagua y sus afluentes. En términos de las divisiones administrativas actuales, correspondía a los municipios de Cali, Jamundí, Dagua, La Cumbre, Restrepo, Yumbo, Vijes y parte de Yotoco. No incluía la banda derecha del Cauca, cuyos pueblos al Sur de Cartago estaban todavía por conquistar y donde Cali nunca hizo efectiva su jurisdicción, ni el sector septentrional del distrio - el de los indios gorrones- por la condena de su encomendero a, entre otras cosas, la pérdida de sus bienes. Tampoco incluía a las tierras de Calima ni aquellas por las cuales corren los ríos que forman el Anchicayá, ya que entonces éstas no hacían parte de la jurisdicción de Cali.

Más adelante, diremos algo sobre los indios de la alta cuenca del río Anchicayá, que en la tasación de 1559, ya agregados a Cali, figuran como "los naturales de la provincia de la montaña", o más brevemente, "los montañeses")

\section{Disposición de la visita}

El censo de 1552 fue dispuesto por el Cabildo de Cali en la sesión del 10 de junio de este año(4). Había sido ordenado más de un año antes, por el licenciado Francisco Briceño juez de residencia y gobernador interino de la gobernación de Popayán, quien había

(4) AGI, Justicia: Leg. 575: f. 1 v, ss. 
nombrado visitadores a Juan del Eastillo y Alonso de Fuenmayor. Pero como lo explica el acto del ayuntamiento, "después de nombrado fue el dicho capitán Alonso de fuenmayor por mandado del dicho señor gobernador a conquistar las provinçias de guachiconu donde al presente reside..(5) y el dicho jhoan del castillo fallesçio destı presente vida". Así las cosas, y ante el inminente regreso a Cali de Briceño (ausente en el Norte de la gobernación), el Cabildo determinó de nombrar otros visitadores para cumplir el mandado.

El Cabildo se componía por entonces de las siguientes personas: Sebastían de Magaña, tesorero y teniente del gobernador; Andrés Moreno, factor y alcalde ordinario; Rodrigo de Villalobos, Antonio Redondo, Baltasar González, Juan López de Pedrosa, Cristobal Quintero y Alonso de Fuenlabrada, regidores; y el escribano Rodrigo Hernández. La nómina de los visitadores fue aprobada de esta manera:

Antonio Redondo y Alonso de Fuenlabrada: visita de los indios de Bichicama, Xamundi (Jamundí), La Balsa y Lile (6), de las encomiendas de Antón Núñez y de Villalobos, y de los grupos llamados Guaales o Guahales. Todo estos estaban en la zoni entre Cali y el límite Sur del distrito.

Juan López de Pedrosa y Francisco Ruíz: visita de Yumbo, Yande, Bitaco, Pepita, Ucache, Locuta, Ylama y Pacara, y la estancia de Mulahaló.

El capitán Pedro López Patiño de Haro y Rodrigo de Villalobos: visita del valle de Lile ("el valle de Antonio Redondo"), de Yçe, Pete, y la quebrada de Juan de Argüello, y de las encomiendas de Quintero, Juan López, Fuenlabrada, Rodrigo Hernández, Francisco Ruíz y Juan de Lara.

Andrés Moreno: visita de los Bixes (Vijes) y los Gorrones.

Escribano de la visita fue nombrado Francisco Romo, quien prestó juramento el 17 de junio.

Cabe anotar que el término "visitar" no significa que los encargados del censo so hicieran presentes en todos los cacicazgos para contar personalmente los indios "de servicio" (7). Por lo general, los visitadores, instalándose en la estancia de un encomendero amigo, hacían venir los caciques de las regiones aledañas a darles relació de los varones útiles de cada tribu o sub-tribu. Dieciocho grupos, algunos de ellos importantes pueblos de la Cordillera, fueron visitados en la misma ciudad de Cali. II sistema no parece conducente a una enumeración precisa, sobre todo en vista de qu los jefes indígenas debieron haberse dado cuenta del objeto de la visita, que era el de

(5) Sobre estas actividades de Fuenmayor, véase Romoli de Avery, RCA, Vol.XI: 1962:252-253

(6) Este no es el Lile grande, llamado también el Valle de Lile, que estaba en la sierra a cine leguas de Cali (Cieza de León, Perú 1: XXVIT), como explicamos en el tex to al aclara algunas denominaciones geograficas del distrito caleño, sino un Lile menor que parece habi correspondido al valle del pequeño rio de este nombre, al Sur de Cali. No fue censado 1552 , al menos no bajo esta designacion, ni figura en la tasacion de 1.559. Andagoya con tribuido mucho a la confusion que ha reinado acerca de Lile (el mayor), pues en su dese de demostrar la unidad de las provincias que pretendia gobernar, y de borrar el recuerdo Belalcazar fundador, ex tendio el nombre de "Lili" no solamente a Cali sino hasta a las tier de la Cordillera al Oriente del Cauca, a las que llamó "Lill de las Sienras". (Relacion de 1541 1546: AGI, Patronato: Leg. 26: Ramo 5, transcitan indios, en Navarrete, Viages: III, y en otros lugares.

(7) La obligación de los visitadores de hacerse presentes en los pueblos que tasaran, fiu decretada por Felipe II en julio de 1552, y aún no era conocida en estas partes de las India al tiempo del censo. Anotamos que todavía en 1559, el oidor López y el obispo Valli pasaron por al to la ordenanza, al hacer la tasación de las encomiendas de la Gobernación Popayán por datos recibidos en las ciudades cabeceras (AGI, Audiencia de Quito: Leg. 60) poder tasar al tributo que se exigía a los encomendados. Sin embargo, la impresión que se saca de los documentos es que los caciques, quizás por orgullo, eran más propensos exagerar el número de sus súbditos, que a disminuírlo. Por otra parte, los españoles valían de sus yanaconas para tener noticias más o menos exactas sobre sus encomiendas.

\section{La ubicación de los pueblos}

Las indicaciones que proporcionan los autos de las visitas y otros documentos de la época, permiten establecer la ubicación de un cierto número de los pueblos con relativa precisión, y de casi todos es posible decir en qué sector del distrito se hallaban.

De acuerdo con esos indicios, hemos dividido el territorio censado en tres zonas, las que con respecto a Cali son: Sur (I), Norte-noreste (II) y Noroeste (III). La Zona I queda exactamente delimitada; la II y la III hasta cierto punto se confunden o se sobreponen, pues no hemos podido situar exactamente en el mapa a varios cacicazgos que en corto lapso desaparecieron.

En esta conexión, cabe aclarar algunos términos que se emplean en los documentos-fuentes. Sabido es que "pueblo", hablando de indios, quería decir tribu o cacicazgo, y por extensión, la tierra perteneciente a éste; y que "provincia" significaba el territorio ocupado por un número de grupos afines y de la misma lengua. Con respecto al distrito de Cali, había dos denominaciones despistadoras: "la provincia de la montaña" y "el valle". La primera no refería todas las partes de la Cordillera Occidental que caían dentro del distrito caleño, sino la región de los ríos cabeceras del Anchicayá y del alto Pepito, cuyos indios, fuertes y dóciles, pagaban el tributo en servicios de cargueros. Y El Valle no era el valle grande del Cauca, como a veces se ha creído, sino el valle de Lile, llamado también el valle de la montaña, que era el del río Bitaco. En 1552, el valle por excelencia, encomienda de Antonio Redondo, parece haber sido la abertura hoy llamada de Pavas. En 1559, sin embargo, ocho pueblos, uno de ellos el de Bitaco, están registrados como "en el valle" (8);1563 los Mercedarios denominaban "Provincia y Doctrina del Valle", o "del valle de la montaña", a toda la cuenca del Dagua y sus tributarios (9)

Los documentos confirman lo dicho por Cieza de León, el único cronista que participó en el descubrimiento y conquista del Occidente de Colombia, quien llegó a Cali con Badillo en noviembre de 1538 , a tres años escasos del primer descubrimiento del territorio: "Los indios y caciques que sirven a los señores que los tienen por encomienda, estan en las sierras" (Perú I: XXVII) Cieza oyó decir que todo el valle del

(8) Si las informaciones que proporcionan las fuentes hacen practicamente segura identificación del Valle de Lile, las de la tasación de 1559 parecen indicar que ya por entonces "El Valle" abarcaba, en el uso español del término, toda la alta cuenca del río Dagua. En el acta de la tasacación, Cendo, Quiguata y Bono se califican como "en el valle". Cendo parece relacionado con el actual Sendo, entre el río Jordan y El Salado; y Quiguata, Conde corren las quebradas de co "la quebrada que divide la dicha loma " (ANHQ. Fondos por clasificar entregados dicha lom (A) "Autos de Dorte Suprema y provenientes de la Audiencia Real: "Autos de Don M contra Don Manuel de la Fuente..."). Anotamos que Petecuy, que en veces se ha querido
identificar con Lile, no era considerado como pueblo del valle.

(9) Monroy, 1930: 44,59 Escobar, en 1582, fu stiga a los Mercedarios de Cali, "padres ydiotas e gente que a diez años que le tienen contra su voluntad al Obispo tomado las doctrinas de 1 a mon taña de Cali e la que llaman del Valle" (AGI, Patronato: Leg. 27; publicado por Jijón y Caamaño, 1938: Apén. 6, y en otros lugares). 
Cauca "fue primero muy poblado de muy grandes y hermosos pueblos, las casas juntel y muy grandes" (op cit.: XXVI), pero lo que vió fue distinto: "En el gran valle de Cal con ser muy fértil, estan las vegas y llanos con su yerba desiertas, y no dan provecho sino a los venados y a otros animales que los pasean, porque los cristianos no son tantos que puedan ocupar tan grandes campiñas". En cuanto a los hermosos pueblos do las "muy grandes provincias llenas de millares de yndios" del valle grande, no sabremo decir. Quizás, los interesantes restos hallados por Bray y Moseley en 1964(10), son evidencia de tales pueblos, ya desaparecidos cuando cieza pasó por allí. Andagoy coincide con Cieza en afirmar que Sebastián de Belalcazar había destruído in floreciente población de los llanos del valle, donde antes había pueblos de 500 y 800 casas, añadiendo que cuando él llegó no había 10.000 hombres "por visitación" en 30 leguas de territorio.

Hay que tener presente, empero, que Cieza, y más Andagoya, miran a hacer resaltar los excesos de Belalcazar. Estos eran en verdad muchos, pero es de dudar que un exiguo número de españoles hubiere podido, en tres o cuatro años, acabar con un población muy numerosa en un terreno tan extenso; especialmente en vista de que so trataba (al decir de Cieza) de gente acostumbrada a guerrear. Los relatos de descubrimiento no hablan sino de un asiento de consideración en el plan al sur del Río Frío: el del cacique Xamundí, que estaba en la orilla del Río Jamundí, algo arriba do llano abierto. En tiempos de Cieza de León, los indios de este cacicazgo vivían "en el nacimiento de este río.... que se extiende tres o cuatro leguas de una parte". Los autos de la visita de 1552 demuestran que varias tribus o sub-tribus vivían en los valles laterales al sur de Cali - no se sabe, en vista de lo dicho por Cieza, qué tan arribaque la de los Guales o Guahales estaba regado entre las cienagas y en las islas que formaba el Cauca en el sector meridional del distrito. Pero aún agregando a todos lo de la zona sur, la de Vijes al Norte (que parece haber estado, al menos en parte, en el plan) no se alcanza sino a 15 o 16 porciento del total de los censados en 1552 .

Por otra parte, la sierra ofrecía condiciones mucho mejores que no la marger izquierda del Cauca. Aquí en mucho trecho había ciénagas y guaduales impenetrables: a más de inundaciones devastadoras en época de las lluvias. En cambio, una ver superada "la sierra baja" - en gran parte estéril- se encuentra entre ésta y "la sierr alta" (las cumbres) una faja verde, fértil, de buenas aguas y de clima inmejorable. Dapa, la parte alta del valle del Chocho, Yumbillo, Santa Inés, pertenecen a esto hermoso sector intermedio, del cual se pasa por collados fáciles a los igualmento favorecidos valles y laderas de la hoya del Dagua y sus tributarios. (Véanse las Lámina 1 a 9 ).

Con respecto a unos topónimos indígenas que aún subsisten en las tierras bajat anotamos que Yumbo, cuyo nombre era también el de una tribu ecuatoriana, ne corresponde al sitio habitado por los Yunbo, o Yumbo, ya que éstos estaban "en la montaña"(11), sino al paraje de una estancia a que dieron este nombre; que Mulahale

(10) Bray y Moseley estudiaron intensivamente un trecho de 23 kilometros, desde tres kilómetro al Norte de Buga hasta veinte al Sur de esta ciudad en el verano de 1964. La investigación "produjo restos de 28 centros de vivienda... La real densidad de población habría sido mucho" may or" y Bray y Moseley creen que todo el fondo del valle grande del Cauca era densamente poblado hasta el tiempo de la conquista española ("An arqueological sequence from the vicinity of Buga, Colombia", en Ñapa Pacha: V. 7-8: p.85 et seq.) Por una opinión diferente, v. Ford, 1944 (11) Declaraciones de Miguel Muñoz y de Doña María de Carbajal, 1551; AGI, Justicia: Leg. 573:
Pza. 3 (hoy Mulaló) era otra estancia, asimismo homónimo de un pueblo y encomienda del Reino de Quito. Cuando la tasación de 1559 habla de los "pueblos" de estas estancias, refiere los encomendados de los respectivos dueños. El toponímico Amaime, distingue actualmente un río y un corregimiento en la banda oriental del Cauca, pero e Amaime de 1552 estaba en la banda occidental y probablemente en la sierra, ya que más tarde fue agregado a Dagua.

Por otro lado, varios nombres autóctonos que hoy existen, no figuran en los documentos de aquel entonces: entre ellos los de Yotoco, Pichindé, Guachinte. E nombre del Río Cauca no es propio de esta parte de Colombia, sino de la del actual Antioquia septentrional. Y Cali, que Castellanos describe -tal vez por las exigencias de la rima- como "prepotente señorío", no existía como tribu o cacicazgo: el nombre es apócope de Calili, denominación de una "provincia" distinta del lugar en que se estableció, por traslado, la ciudad española de Cali (12).

\section{El aspecto demográfico}

Evidentemente, la importancia de los datos que proporciona el censo de 1552 , no es solamente lingüística y clasificadora. El empadronamiento nos brinda la primera información segura de orden demográfico respecto a esta región de Colombia información que resulta particularmente significativa con relación a la que dan la tasación de 1559 y unas estimaciones posteriores de la población indígena.

Según una ordenanza del rey Felipe II, promulgada en 1578 (Leyes: Lib.VI: tit. 5; ley VII), hasta esta fecha los tributarios eran solamente los hombres casados. No obstante, al cotejar los datos de la visita de 1552 y los de la tasación de 1559 (13), está claro que se consideraban como útiles a todos los varones adultos. En la tasación, sin embargo, se especifica el número de casados y el de solteros en cada pueblo de encomienda. En las zonas que habían sido empadronadas en 1552 -las que aquí nos interesan- había al tiempo de la tasación, una total de 2.343 tributarios, de los cuales 1.577 eran hombres casados (familias). Suponiendo que la ecuación de casados a solteros (2,06: 1, o 32,69 por ciento de solteros) fuese la misma al tiempo del empadronamiento, había entonces en el área censada 2.252 hombres casados. La merma en el número de familias que se obró en los siete años que transcurrieron entre las dos visitas, fue de aproximadamente 30 por ciento.

(Aproximadamente, porque aunque el área en consideración es la misma, no coinciden sino en parte los elencos de pueblos de las dos visitas. En 1559 , se tasaron, tanto unos cacicazgos mencionados pero no censados en 1552 como otros siete no antes nombrados; mientras que 24 de los pueblos visitados en 1552 habían desaparecido del registro. De éstos, cinco o seis pueden corresponder a otros tantos de los "nuevos", por haberse cambiado el cacique por cuyo apellido se denominaba el grupo; los otros se

(12) Calili está mencionado en varios documentos del tiempo de la conquista. No figura en nuestras listas de nombres, por ser ajeno a las regiones censadas en 1552

(13) Los autos oidor Tomás López de la Audiencia de Santa Fe del Nuevo Reino con la asesoría del obispo Valle en toda la Gobernación de Popayán, estan en AGI. Sección Justicia: Audiencia de Quito: Legajo 60. Refieren los pueblos encomendados, a veces por un toponímico, a veces por un gentilicio o por el apellido del cacique respectivo, con el número de tributarios, tanto casados como solteros, en cada uno de ellos; y fijan el tributo debido, en especies y servicios, en cada encomienda. Friede reporta los datos de la tasación en su interesante libro sobre el obispo Valle (1961, cap. XIX), con algunas equivocaciones -muy comprensibles- de copia e in terpretación. 
extinguieron o, ya reducidos, fueron agregados a cacicazgos distintos. En el cuadro comparativo (Tabla 5), se ven estas modificaciones.

Las estimaciones de la población indigena -más exactamente, de la cuantía de hombres de mayor edad en esta población- que se encuentran en documentos posteriores a 1559 , se refieren en números redondos a todo el distrito, inclusos la provincia de la montaña y el sector septentrional. Hacia 1572-1574, pero al parecer con base en informes bastante anteriores, López de Velasco la calculó en 3.000; en 1582, Fray Jerónimo Escobar, quien estaba bien informado de las cosas de la gobernación, la puso en 2.000; en 1634 el gobernador Villaquirán dijo, en un informe oficial (14), que sumaban 420. Si a las listas de 1552 agregamos los montañeses, en número correspondiente a la proporción de estos al total que presenta la ecuación de 1559, y, si las estimaciones más atendibles son razonablemente verosímiles, el cuadro de los indios útiles sería aproximadamente así:

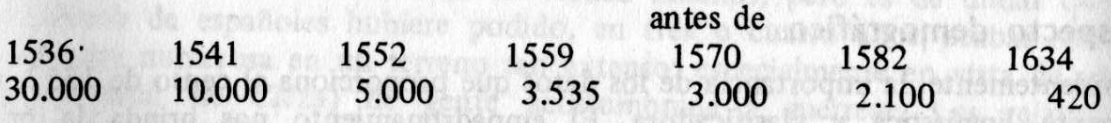

\section{El Padrón}

El Padrón General de los varones adultos censados en 1552, que presentamos a continuación, registra todos los apellidos contenidos en los roles de los 62 grupos visitados, con excepción de los nombres españoles (61, para los cuales no hay contranombre indígena) y los apodos puestos por españoles (Pocaropa, Caballo, Cucaracha, etc.), que son ocho o nueve (15). Como ya anotamos, los individuos empadronados están identificados por las zonas, pueblos y caciques respectivos.

En la transcripción, hemos respetado fielmente la ortografía de los originales. Es bueno tener presente, sin embargo, tanto el hecho de que los escribientes recibieron los datos, en la mayoría de los casos a través o de interpretes yanaconas, como las peculiaridades de la fonética castellana de aquel entonces, cuando algunas consonantes tenían valores diferentes de los modernos. La $j$ era suave ( $j$ francesa), la $x$ tenía sonido de sh inglesa (s), la ç de ts y la $z$ de ds. Es evidente que mientras estos sonidos podían a veces facilitar la transcripción de vocablos exóticos, se prestaban también a trueques entre sí. Con todo, unas cuidadosas correcciones hechas por los escribientes en ciertos apellidos, demuestra que se hizo lo posible para que las formas fuesen registradas de manera exacta.

Para facilitar la consulta del nomenclador, he aquí la lista de los topónimos y gentilicios que registran los autos de la visita de 1552

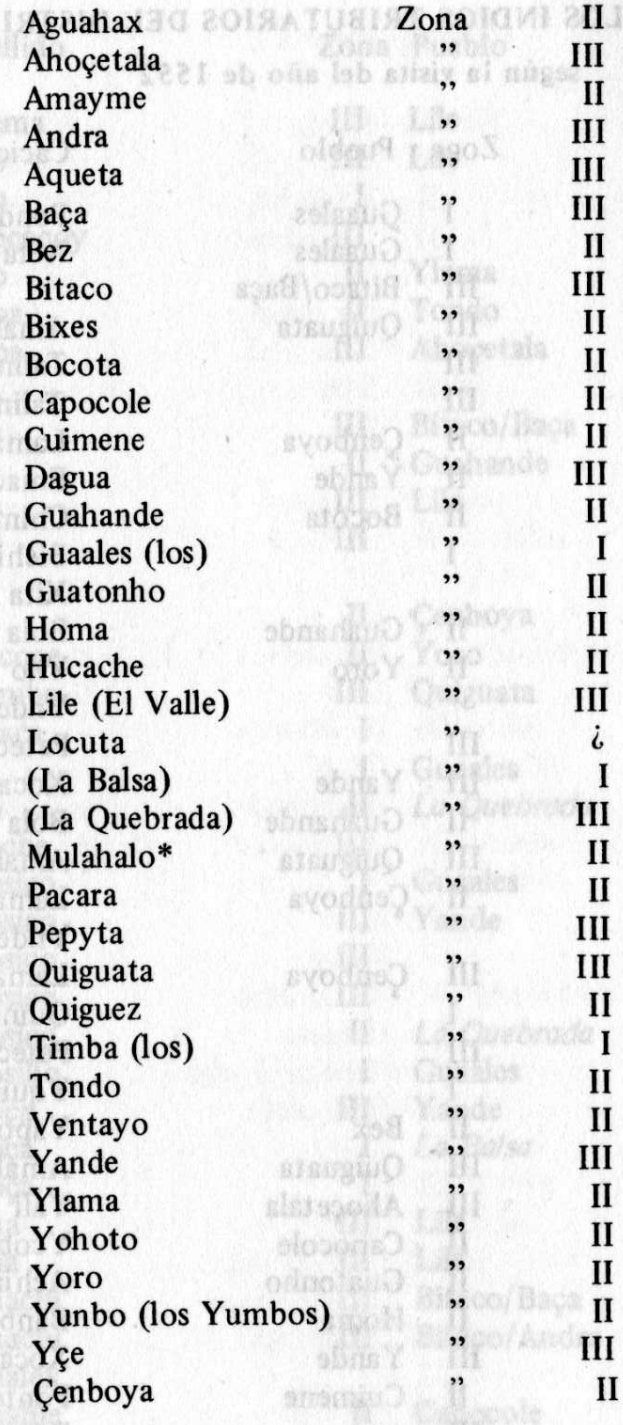

*Al parecer, nombre importado del Ecuador.

(14) Transcrito en Proaño Mss: Tomo III: f. 4-13.

(15) Hay otros dos apellidos que sugieren un origen español: Gavitia, que se podría imaginar un error, por Gaviria; y Tamayo, que es apellido de conquistador, pero a la vez netamente indígena. Dos caciques se identificaron con un nombre cristiano en adición al apellido indígena, y dos indios tenían moquetes españoles a la vez de apellidos nativos. 


\begin{tabular}{|c|c|c|}
\hline ona & Pueblo & Cacique \\
\hline I & Guaales & Ponde \\
\hline 1 & Guaales & Xeta \\
\hline III & Bitaco/Baça & \\
\hline III & Quiguata & Amalile \\
\hline III & & Talima \\
\hline III & & Talima \\
\hline II & Çenboya & Lama \\
\hline II & $\overrightarrow{\mathrm{Y}}$ ande & Emacui \\
\hline II & Bocota & Chimba \\
\hline I & 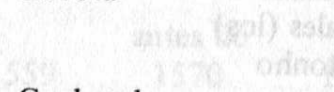 & $\begin{array}{l}\text { Bichicama } \\
\text { Xita }\end{array}$ \\
\hline II & Guahande & Bola \\
\hline II & Yoro & $\begin{array}{l}\text { Yho } \\
\text { Yndegua }\end{array}$ \\
\hline III & & Petecui \\
\hline III & Yande & Xocate \\
\hline II & Guahande & Bola \\
\hline III & Quiguata & Amalile \\
\hline II & Çenboya & $\begin{array}{l}\text { Lama } \\
\text { Yndegua }\end{array}$ \\
\hline III & Çenboya & Lama \\
\hline I & & Quincha \\
\hline III & & Petecui \\
\hline I & & Yquinde \\
\hline II & Bex & Yapo \\
\hline III & Quiguata & Amalile \\
\hline III & Ahoçetala & Yali \\
\hline II & Capocole & Ycobale \\
\hline II & Guatonho & Achindeta \\
\hline II & Homa & Banbayaco \\
\hline III & Yande & Xocate \\
\hline II & Cuimene & Pontecui \\
\hline III & & Petecui \\
\hline III & & Talima \\
\hline & aryogaris stat & Onze \\
\hline III & & Chumba \\
\hline III & & Talima \\
\hline III & & Petecui \\
\hline III & & Çendo \\
\hline III & & Petecui \\
\hline III & & Pondo \\
\hline II & Guatonho & Achindeta \\
\hline III & & Talima \\
\hline
\end{tabular}

$\begin{array}{ll}44 & \text { Ahema } \\ 45 & \text { Aho } \\ 46 & \text { Aho } \\ 47 & \text { Ahoçecoy } \\ 48 & \text { Ailo } \\ 49 & \text { Ajipa } \\ 50 & \text { Ajipa } \\ 51 & \text { Ajo } \\ 52 & \text { Ajo } \\ 53 & \text { Ajo } \\ 54 & \text { Ajo } \\ 55 & \text { Ajo } \\ 56 & \text { Ala } \\ 57 & \text { Ala } \\ 58 & \text { Alacoçe } \\ 59 & \text { Alambe } \\ 60 & \text { Alcaça } \\ 61 & \text { Ali } \\ 62 & \text { Alo } \\ 63 & \text { Aloche } \\ 64 & \text { Alosico } \\ 65 & \text { Alosico } \\ 66 & \text { Alosico } \\ 67 & \text { Alosico } \\ 68 & \text { Alosico } \\ 69 & \text { Alosillo } \\ 70 & \text { Aloça } \\ 71 & \text { Aloça } \\ 72 & \text { Aloça } \\ 73 & \text { Ama } \\ 74 & \text { Ama } \\ 75 & \text { Amacui } \\ 76 & \text { Amacui } \\ 77 & \text { Amalex } \\ 78 & \text { Amalile } \\ 79 & \text { Amalile } \\ 80 & \text { Amalile } \\ 81 & \text { Amalile } \\ 82 & \text { Amandine } \\ 83 & \text { Amano } \\ 84 & \text { Amanoa } \\ 85 & \text { Amanoa } \\ 86 & \text { Amanta } \\ 87 & \text { Amanta } \\ 89 & \text { Amanta } \\ & \text { Amba }\end{array}$

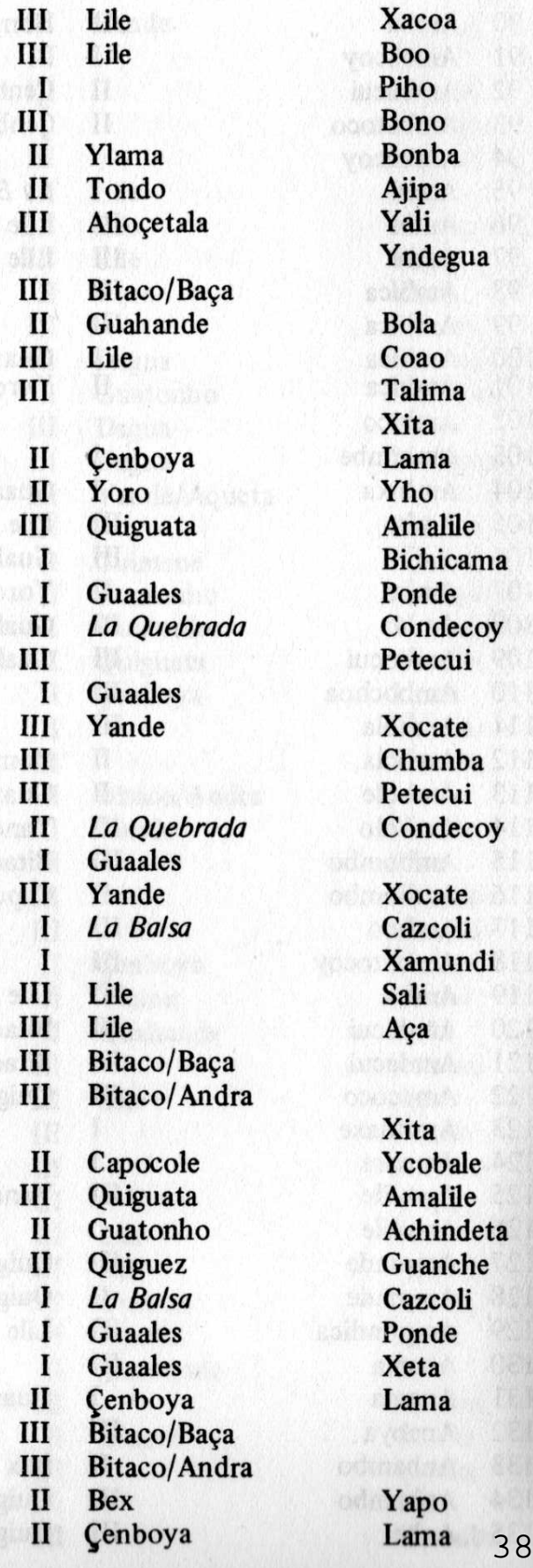


II Homa

I

II Çenboya

II Çenboya

94 Ambecoy

95 Ambi

96 Ambi

97 Ambi

98 Ambica

99 Ambica

100 Ambica

101 Ambica

102 Ambico

103 Ambimbe

104 Ambixa

105 Ambo

106 Ambo

107 Ambo

108 Ambo

109 Ambocui

110 Ambochoa

111 Ambola

112 Ambola

113 Ambole

114 Ambolo

115 Ambombo

116 Ambombo

117 Amboo

118 Ambotocoy

119 Ambu

120 Amdacui

121 Amdacui

22 Amecoco

23 Amitoaxe

24 Amoara

25 Amolile

26 Amolile

127 Ampinde

129 Ampondica

130 Anama

131 Anama

132 Anaoya

133 Anbambo

134 Anbambo

III Lile

III Lile

III

I Guaales

II Yoro

I

III Lile

III Guahande

II Yoro

III Guahande

III

II Homa

II Guatonho

III Yande

II Capocole

III

III. Lile

III Quiguata

I

III Quiguata

II Quiguez

III Lile

I Guaales

II Bex

III Quiguata

Banbayaco
Yquinde
Lama
Lama
Chiguatoco
Cazcoli
Xacoa
Boo
Talima
Pondo
Ponde
Yho
Onze
Quincha
Ponde
Xacoa
Bola
Yho
Bola
Bola
Chumba
Petecui
Banbayaco
Achindeta
Diacuy
Ycobale
Petecui
Petecui
Boo

Amalile
Xamundi
Quincha
Cayoyo
Amalile
Guanche
Coao
Petecui
Xeta
Pondo
Yapo
Amalile
Amalile

$\begin{array}{ll}136 & \text { Anbimbe } \\ 137 & \text { Anbimbi } \\ 138 & \text { Anboesto } \\ 139 & \text { Ançe } \\ 140 & \text { Anchicui } \\ 141 & \text { Ancho } \\ 142 & \text { Ancho } \\ 143 & \text { Ancho } \\ 144 & \text { Anda } \\ 145 & \text { Andacoy } \\ 146 & \text { Andacui } \\ 147 & \text { Andacui } \\ 148 & \text { Andacui } \\ 149 & \text { Andacui } \\ 150 & \text { Andagua } \\ 151 & \text { Andama } \\ 152 & \text { Andapa } \\ 153 & \text { Andapa } \\ 154 & \text { Andaqua } \\ 155 & \text { Andaquindex } \\ 156 & \text { Andaxa } \\ 157 & \text { Andaça } \\ 158 & \text { Andaçe } \\ 159 & \text { Andica } \\ 160 & \text { Ando } \\ 161 & \text { Ando } \\ 162 & \text { Ando } \\ 163 & \text { Ando } \\ 164 & \text { Ando } \\ 165 & \text { Ania } \\ 166 & \text { Anima } \\ 167 & \text { Anima } \\ 168 & \text { Anqui } \\ 169 & \text { Anto } \\ 170 & \text { Anto } \\ 171 & \text { Anto } \\ 172 & \text { Anto } \\ 173 & \text { Anzo } \\ 174 & \text { Aoa } \\ 175 & \text { Aotaque } \\ 176 & \text { Aotax } \\ 177 & \text { Aoxe } \\ 178 & \text { Aoylo } \\ 179 & \text { Aoyna } \\ 180 & \text { Aoyne } \\ 181 & \text { Apa }\end{array}$

II Yande

Emacui

Bono

Petecui

Yndegua

Talima

Boo

III Lile

I Guaales

III Guahande

III Bitaco/Andra

III Bitaco/Baça

III Bitaco/Baça

III Yande/Aqueta
III Lile

Xacoa

Sali

Yho

Petecui

Benbacui

Achindeta

Ypiçe

Benbacui

Petecui

Pontecui

Achindeta

Achindeta

Amalile

Lama

Talima

Petecui

Xocate

Piho

Talima

Petecui

Lama

I Çenboya

II Ylama

II Guahande

Bonba

Bola

Petecui

III Lile

III

III

III

III Lile

III Lile

I Guaales

II Tondo

III

II Ylama

Aça

Pondo

Talima

Çendo

Boo

Aça

Xeta

Ajipa

Bola

Petecui

Bonba

Çendo

Xita

181 Apa
II Guahande 


$\begin{array}{ll}182 & \text { Apa } \\ 183 & \text { Apale } \\ 184 & \text { Apandica } \\ 185 & \text { Apanteca } \\ 186 & \text { Apata } \\ 187 & \text { Apate } \\ 188 & \text { Apayali } \\ 189 & \text { Ape } \\ 190 & \text { Ape } \\ 191 & \text { Apenco } \\ 192 & \text { Apiisque } \\ 193 & \text { Apito } \\ 194 & \text { Aquehe } \\ 195 & \text { Aques } \\ 196 & \text { Aqui } \\ 197 & \text { Aquile } \\ 198 & \text { Aquinba } \\ 199 & \text { Aquinde } \\ 200 & \text { Aquinçe } \\ 201 & \text { Aquipaya } \\ 202 & \text { Aquistaça } \\ 203 & \text { Aquiçe } \\ 204 & \text { Aquiçe } \\ 205 & \text { Aquiz } \\ 206 & \text { Aquiz } \\ 207 & \text { Aquo } \\ 208 & \text { Aquta } \\ 209 & \text { Atame } \\ 210 & \text { Atame } \\ 211 & \text { Atanche } \\ 212 & \text { Atanche } \\ 213 & \text { Atandica } \\ 214 & \text { Atanoa } \\ 215 & \text { Atende } \\ 216 & \text { Atende } \\ 217 & \text { Atende } \\ 218 & \text { Atende } \\ 219 & \text { Atende } \\ 220 & \text { Atende } \\ 221 & \text { Atende } \\ 222 & \text { Atende } \\ 223 & \text { Atende } \\ 224 & \text { Atende } \\ 225 & \text { Atende } \\ 226 & \text { Atende } \\ 227 & \text { Atende } \\ 88 & \end{array}$

\begin{tabular}{|c|c|c|}
\hline III & Lile & Boo \\
\hline II & Quiguez & Guanche \\
\hline II & Tondo & Ajipa \\
\hline II & Ventayo & Taqui \\
\hline III & Quiguata & Amalile \\
\hline III & & Chumba \\
\hline III & Quiguata & Amalile \\
\hline II & Çenboya & $\begin{array}{l}\text { Lama } \\
\text { Yndegua }\end{array}$ \\
\hline III & in & Talima \\
\hline III & & Petecui \\
\hline II & Guatonho & Achindeta \\
\hline II & La Quebrada & Condecoy \\
\hline II & Guahande & Bola \\
\hline III & Quiguata & Amalile \\
\hline III & & Petecui \\
\hline III & & Talima \\
\hline II & Çénboya & Lama \\
\hline II & Ventayo & Taqui \\
\hline III & & Petecui \\
\hline I & Guaales & Monaco \\
\hline II & Bocota & Chimba \\
\hline II & Çenboya & Lama \\
\hline III & Dagua & Benbacui \\
\hline III & Lile & Coao \\
\hline II & Çenboya & Lama \\
\hline II & Capocole & Ycobale \\
\hline II & Guahande & Bola \\
\hline III & & Çendo \\
\hline I & & Bichicama \\
\hline I & & Bichicama \\
\hline I & & Bichicama \\
\hline I & & Xamundi \\
\hline & & Yndegua \\
\hline III & Bitaco/Andra & 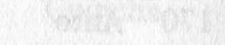 \\
\hline III & Bitaco/Andra & $(x+2)=$ \\
\hline III & Yande & Diacuy \\
\hline III & Yande & Diacuy \\
\hline III & & Pondo \\
\hline III & & Talima \\
\hline III & & Talima \\
\hline III & Lile & Coao \\
\hline III & & Petecui \\
\hline III & & Petecui \\
\hline & & Xita \\
\hline & & Chiguatoc \\
\hline
\end{tabular}

$\begin{array}{ll}228 & \text { Atende } \\ 229 & \text { Atende } \\ 230 & \text { Atende } \\ 231 & \text { Atende } \\ 232 & \text { Atende } \\ 233 & \text { Atende } \\ 234 & \text { Atende } \\ 235 & \text { Atendechico } \\ 236 & \text { Atimbo } \\ 237 & \text { Atoco } \\ 238 & \text { Atonche } \\ 239 & \text { Atonde } \\ 240 & \text { Atoni } \\ 241 & \text { Atonçe } \\ 242 & \text { Atonza } \\ 243 & \text { Au.ys } \\ 244 & \text { Aua } \\ 245 & \text { Aucama } \\ 246 & \text { Aucana } \\ 247 & \text { Avita } \\ 248 & \text { Axacoe } \\ 249 & \text { Axala } \\ 250 & \text { Axaque } \\ 251 & \text { Axda } \\ 252 & \text { Axera } \\ 253 & \text { Axicui } \\ 254 & \text { Axicui } \\ 255 & \text { Axo } \\ 256 & \text { Axo } \\ 257 & \text { Axo } \\ 258 & \text { Axo } \\ 259 & \text { Axo } \\ 260 & \text { Axocui } \\ 261 & \text { Axopelo } \\ 262 & \text { Axota } \\ 263 & \text { Axumbo } \\ 264 & \text { Ayagua } \\ 265 & \text { Ayazagua } \\ 266 & \text { Ayoz } \\ 267 & \text { Ayuchi } \\ 268 & \text { Aça } \\ 269 & \text { Açalua } \\ 270 & \text { Açendeta } \\ 271 & \text { Açençe } \\ 272 & \text { Azcui } \\ 273 & \text { Azombo } \\ & \\ & \end{array}$

\begin{tabular}{|c|c|c|}
\hline II & Cuimene & Pontecui \\
\hline II & Cuimene & Pontecui \\
\hline II & Guatonho & Achindeta \\
\hline II & Guatonho & Achindeta \\
\hline II & Guatonho & Achindeta \\
\hline II & Guatonho & Achindeta \\
\hline II & Bocota & Chimba \\
\hline III & Bitaco/Andra & \\
\hline II & La Quebrada & Condecoy \\
\hline II & Guatonho & Achindeta \\
\hline III & & Peticui \\
\hline II & Ventayo & Taqui \\
\hline II & La Quebrada & Xequino \\
\hline II & Capocole & Ycobale \\
\hline III & Ahocetala & Yali \\
\hline II & Guahande & Bola \\
\hline II & Guahande & Bola \\
\hline III & Lile & Boo \\
\hline III & Lile & Xacoa \\
\hline III & & Chumba \\
\hline II & Cuimene & Pontecui \\
\hline II & Cuimene & Pontecui \\
\hline III & Quiguata & Amalile \\
\hline III & Quiguata & Amalile \\
\hline III & & Talima \\
\hline II & Yohoto & Yqua \\
\hline II & Yohoto & Yqua \\
\hline II & Senboya & Lama \\
\hline II & Guahande & Bola \\
\hline II & Bocota & $\begin{array}{l}\text { Chimba } \\
\text { Onze }\end{array}$ \\
\hline III & Bitaco/Andra & \\
\hline II & Bocota & Chimba \\
\hline II & Çenboya & Lama \\
\hline II & Bocota & Chimba \\
\hline III & Yande/Aqueta & \\
\hline I & & Bichicama \\
\hline II & Ylama & Bonba \\
\hline III & Ahoçetala & Yali \\
\hline III & & Petecui \\
\hline III & Lile & Aça \\
\hline II & La Quebrada & Condecoy \\
\hline II & Cuimene & Pontecui \\
\hline III & Quiguata & Amalile \\
\hline III & Yande & Quindecui \\
\hline II & Ventayo & Taqui \\
\hline
\end{tabular}




\begin{tabular}{rll} 
III & \\
III & Yande \\
& \\
I & La Balsa \\
I & \\
I & \\
III & \\
III & \\
II & Capocole \\
II & Guatonho \\
III & Bitaco/Baça \\
III & Lile \\
III & Yande \\
III & Yande \\
III & Bitaco/Andra \\
II & Bocota \\
II & Quiguez \\
II & Yoro \\
III & Yande \\
III & Bitaco/Baça \\
III & Yande \\
III & Quiguata \\
II & Cuimene \\
II & Homa \\
I & \\
I & Guales \\
II & La Quebrada \\
III & Lile \\
II & Bocota \\
III & \\
II & Ylama \\
III & Lile \\
III & Lile \\
III & Lile \\
I & Guaales \\
III & \\
I & \\
III & \\
II & Ventayo \\
III & \\
III & Quiguata \\
I & Guaales \\
II & Bocota \\
III & Dagua \\
III & Bitaco/Andra \\
II & Homa \\
& \\
\hline
\end{tabular}

$\begin{array}{lll}\text { Talima } & 320 & \text { Bencacui } \\ \text { Emacui } & 321 & \text { Bencui } \\ \text { Yndegua } & 322 & \text { Bepo } \\ \text { Cazcoli } & 323 & \text { Bequi } \\ \text { Bichicama } & 324 & \text { Bequi } \\ \text { Monaco } & 325 & \text { Beta } \\ \text { Petecui } & 326 & \text { Betex } \\ \text { Talima } & 327 & \text { Bex } \\ \text { Ycobale } & 328 & \text { Bey } \\ \text { Achindeta } & 329 & \text { Beya } \\ & 330 & \text { Beya } \\ \text { Sali } & 331 & \text { Beye } \\ \text { Diacuy } & 332 & \text { Beçane } \\ \text { Quindecui } & 333 & \text { Bia } \\ & 334 & \text { Bia } \\ \text { Chimba } & 335 & \text { Bia } \\ \text { Guanche } & 336 & \text { Bibepe } \\ \text { Yho } & 337 & \text { Bica } \\ \text { Diacuy } & 338 & \text { Biche } \\ & 339 & \text { Bichicama } \\ \text { Quindecui } & 340 & \text { Bichicama } \\ \text { Amalile } & 341 & \text { Bila } \\ \text { Pontecui } & 342 & \text { Bila } \\ \text { Banbayaco } & 343 & \text { Bilaqui } \\ \text { Piho } & 344 & \text { Bile } \\ \text { Ponde } & 345 & \text { Bilicui } \\ \text { Condecoy } & 346 & \text { Bilo } \\ \text { Aça } & 347 & \text { Biloo } \\ \text { Chimba } & 348 & \text { Bimbe } \\ \text { Pondo } & 349 & \text { Bimbe } \\ \text { Bonba } & 350 & \text { Bimbi } \\ \text { Coao } & 351 & \text { Bime } \\ \text { Xacoa } & 352 & \text { Bina } \\ \text { Coao } & 353 & \text { Binza } \\ \text { Xeta } & 354 & \text { Bipi } \\ \text { Bono } & 355 & \text { Biquina } \\ \text { Bichicama } & 356 & \text { Biquino } \\ \text { Talima } & 357 & \text { Biquino } \\ \text { Taqui } & 358 & \text { Bita } \\ \text { Petecui } & 359 & \text { Bita } \\ \text { Amalile } & 360 & \text { Bitaco } \\ \text { Xeta } & 361 & \text { Bitacui } \\ \text { Chimba } & 362 & \text { Bitaque } \\ \text { Benbacui } & & \\ \text { Banbayaco } & 363 \text { itaque } \\ & \\ & & \\ & & \end{array}$

III Bitaco/Baça

III Lile

II La Quebrada

III Lile

II La Quebrada

II Cenboya<smiles>[AlH2]</smiles>

III

III

I Guaales

III Yande

III Ahoçetala

III

III Yande

III Lile

III

II

III

II Cenboya

III Yande

III Ahoçetala

III Dagua

I

III Yande

I Guaales

II Guatonho

II Cenboya

II Aguahax

III Lile

III ,

II Guatonho

I

I Guaales

III Bitaco/Baça

III Yande

II Guatonho

II Ventayo

II Tondo

II Yohoto
Xacoa

Xepia

Xequino

Aça

Xamundi

Condecoy

Lama

Yquinde

Xamundi

Pondo

Petecui

Xeta

Quindecui

Yali

Chumba

Xocate

Bono

Coao

Petecui

Bichicama

Petecui

Lama

Xocate

Yali

Ypiçe

Quincha

Pondo

Emacui

Achindeta

Lama

Pondo

Yçando

Boo

Petecui

Achindeta

Quincha

Chichino

Petecui

Quindeçui Achindeta

Ajipa

Yqua 
369 Biçe

370 Biçe

371 Biçe

II Guatonho

III Yande

II Senboya

II Cenboya

III

II Bocota

III Bitaco/Andra

I Guaales

II Bocota

III Lile

II Homa

\section{III}

II Guatonho

II Guatonho

I

I

I

I Guaales

II La Quebrada

III

III Lile

II Yohoto

III Quiguata

III Quiguata

III Dagua

III Yande

II Guahande

III Lile

I Guaales

III

III

$\begin{aligned} \text { III } & \text { Ahoçetala } \\ \text { III } & \text { Bitaco/Baça } \\ \text { II } & \text { Guatonho } \\ \text { II } & \text { Aguahax } \\ \text { II } & \text { Tondo } \\ \text { II } & \text { Ylama } \\ \text { III } & \text { Yande } \\ \text { II } & \text { Ylama } \\ \text { II } & \text { Guatonho }\end{aligned}$

Ponde Achindeta Diacuy

Lama

Lama

Chumba

Chumba

Chimba

Monaco

Chimba

Xacoa

Banbayaco

Yndegua

Achindeta

Achindeta

Quincha

Piho

Yquinde

Xeta

Condecoy

Talima

Xacoa

Yqua

Amalile

Amalile

Benbacui

Diacuy

Yqua

Bola

Aça

Monaco

Petecui

Çendo

Chiguatoco

Onze

Yali

Achindeta

Yçando

Ajipa

Bonba

Diacuy

Bonba

Achindeta

\section{Zona Pueblo}

III

$\begin{array}{ll}412 & \text { Bombi } \\ 413 & \text { Bombo }\end{array}$

414 Bombo

415 Bombo

416 Bombo

417 Bombo

418 Bombo

419 Bombo

420 Bona

421 Bonba

422 Bonba

423 Bonba

424 Bonba

425 Bonihe

426 Bono

427 Bono

429 Bono

430 Bono

431 Bono

432 Boo

433 Boo

434 Boo

435 Booy

436 Bopa

437 Boque

438 Bos

439 Boscoy

440 Bota

441 Botacu

443 Boya

444 Boçe

445 Boço

$446 \mathrm{Boz}$

447 Boz

448 Boz

$449 \mathrm{Boz}$

450 Bozcoy

451 Bozcui

452 Bubuye

453 Bucha

454 Buche

455 Bula

456 Bula

457 Buli
III Amayme

II Guatonho

II Guatonho

II Yoro

I La Balsa

I

II Bitaco/Baça

II Ylama

II Tondo

III Lile

III Lile

III Ahoçetala

III

II Ylama

II Homa

II Çenboya

III Yande

III Lile

III Lile

III

I La Balsa

II Guatonho

III

III

II Quiguez

III

II Bocota

III Lile

I Guaales

II Ylama

II Cuimene

II La Quebrada

III

II Ylama

II

I Guaales

II Cenboya

II Yohoto

III Yande
Cacique

Talima

Talima

Yambo

Achindeta

Achindeta

Yho

Cazcoli

Xamundi

Piho

Bonba

Ajipa

Boo

Coao

Yali

Bono

Bonba

Banbayaco

Lama

Emacui

Boo

Aça

Yndegua

Çendo

Cazcoli

Achindeta

Petecui

Petecui

Chiguatoco

Guanche

Petecui

Chimba

Aça

Monaco

Achindeta

Bonba

Pontecui

Xequino

Petecui

Bonba

Petecui

Xeta

Lama

Yqua

Diacuy

Coao 
458 Buna

459 Buocui

460 Buço

461 Caaçe

462 Cabani

463 Cabanica

464 Cabanica

465 Caca

466 Cacui

467 Cache

468 Cache

469 Cagua

470 Cagua

471 Caha

472 Cahancoy

473 Cahancu

474 Cahancui

475 Cahaq

476 Cahecui

477 Cahoho

478 Cahoho

479 Cahoho

480 Cahoho

481 Cahoho

482 Cala

483 Calabo

484 Calambe

485 Calanoa

486 Calembo

487 Cali

488 Calima

489 Calima

490 Calmis

491 Calo

492 Calu

493 Calumbi

494 Cama

495 Cama

496 Cama

497 Cama

498 Cama

500 Cama

501 Camaco

502 Camba
Onze

Amalile

Quiguat

Coao

III Lile

III

I

I

III

III

III

II Çenboya

III Lile

II Yoro

II Guahande

II Quiguata

II Guahande

II La Quebrada

III

Aça

Petecui

Xamundi

Xamundi

Sendo

Pondo

Petecui

Cayoyo

Lama

Xacoa

Xita

Bola

Xita

Condecoy

Petecui

Onze

Xita

III Amayme

I Guaales

II Çenboya

II Yoro

II Guahande

II Cenboya

I Guaales

II La Quebrada

III Lile

II Bex

II La Quebrada

III Ahoçetala

III Lile

II Guahande

II La Quebrada

III Yande
503 Cambacui

504 Cambe

505 Cambecui

506 Cambo

507 Cambo

508 Cambo

509 Cambo

510 Cambo

511 Came

512 Cameta

513 Camo

514 Camocola

515 Canayoa

516 Canbaca

517 Canbala

518 Cancha

519 Cancha

520 Canchagua

521 Cancho

522 Canda

523 Canda

524 Candacui

525 Cande

526 Candi

527 Candia

528 Candimula

529 Candipa

530 Cando

531 Cane

532 Canegua

533 Cangua

534 Cani

535 Cani

536 Cani

537 Canima

538 Cano

539 Canoa

540 Canoco

541 Canoma

542 Canoma

543 Canoma

544 Canomacal

545 Canqui

546 Canta

547 Canta

548 Cantacui
III Quiguata

II Çenboya

II Yoro

II Homa

II Çenboya

II Bocota

II Ylama

II Tondo

II Bocota

III Lile

III

II Guahande

I La Balsa

III Lile

I Guaales

III Bitaco/Andra

I Guaales

I Guaales

I Guaales

II La Quebrada

II Çenboya

II La Quebrada

III Lile

III

I La Balsa

III Lile

II La Quebrada

III

III

II Guahande

III Lile

III

II La Quebrada

III

III

II La Quebrada

Guaales

Guaales

I

III Lile

III Lile

III Lile

III Yande
Amalile

Lama

Yho

Banbayaco

Lama

Chimba

Bonba

Ajipa

Chimba

Boo

Chumba

Bola

Cazcoli

Boo

Ponde

Ponde

Chichino

(Alonso)

Yquinde

Xequino

Lama

Xequino

Boo

Petecui

Cazcoli

Boo

Condecoy

Çendo

Petecui

Bola

Boo

Yndegua

Talima

Xamundi

Xequino

Çendo

Çendo

Xequino

Monaco

Monaco

Xepia

Xacoa

Boo

Aça

Diacuy 
549 Ċanuiçala

550 Cançecui

551 Canza

552 Canzacoy

553 Canzica

554 Caoa

555 Caoc

556 Caoende

557 Caota

558 Capa

559 Capa

560 Capegoc

561 Capença

562 Capez

563 Capi

564 Capito

565 Capita

566 Carani

567 Caribe

568 Caspa

569 Catama

570 Catane

571 Catane

572 Catane

573 Catani

574 Catani

575 Catani

576 Cate

577 Catheme

578 Cati

579 Cato

580 Catole

581 Catole

582 Cauaque

583 Cavy

584 Caxcu

585 Cay

586 Cayamiçe

587 Cayo

588 Cayo

589 Cayo

590 Cayocui

591 Cayocui

592 Cayondix

$$
\begin{aligned}
\text { II } & \text { Guahande } \\
\text { I } & \text { La Balsa }
\end{aligned}
$$

III Lile

II La Quebrada

I Guaales

II La Quebrada

II Yoro

II Ventayo

I Guaales

II Ylama

II Guahande

II Cenboya

II Guatonho

III Quiguata

II Cenboya

II La Quebrada

III Lile

II Tondo

II Bocota

II Bocota

III Lile

II La Quebrada

II La Quebrada

III Lile

II Bocota

II Cenboya

III Lile

II Yoro

II Yoro

II Yande

II Çenboya

II Capocole

III

I La Balsa

II Guahande

III Lile

II Çenboya

II Guahande

II Bocota
Xamundi

Bola

Cazcoli

Petecui

Sali

Xequino

Ponde

Xequino

Yho

Taqui

Chichino

Bonba

Talima

Bola

Lama.

Achindeta

Amalile

Yndegua

Lama

Condecoy

Aça

Ajipa

Chimba

Chimba

Aça

Condecoy

Condecoy

Boo

Chimba

Lama

Sali

Yho

Yho

Quindecui

Lama

Ycobale

Bono

Cazcoli

Petecui

Bola

Xacoa

Lama

(4) Bola

Chimba
594 Caçaque

595 Caçe

596 Caçiguaya

597 Caçumba

$598 \mathrm{Caz}$

599 Cazcoli

600 Cazcui

601 Celemiche

602 Coa

603 Coa

604 Coa

605 Coaco

606 Coaho

607 Coambi

608 Coao

609 Cobe

610 Cocaho

611 Cocatama

612 Cocaçi

613 Coco

614 Cocolama

615 Cocoo

616 Cocucuy

617 Cocute

618 Coche

619 Coche

620 Cochii

621 Cocho

622 Cocho

623 Cocho

624 Coecoe

625 Coeho

626 Coeho

627 Coemi

628 Coençecoy

629 Coexa

630 Coeçe

631 Coez

632 Coez

633 Coheho

634 Coheto

635 Cohinche

636 Coho

637 Coho

638 Cohonco

639 Cohoo
III

I

II La Quebrada

II Bocota

I La Balsa

II Cuimene

II Ylama

II Guahande

III Lile

III

III

III Lile

III Ahoçetala

I

I

II Cuimene

I La Balsa

III Lile

I Guaales

III Lile

III Lile

III

I Guaales

I Guaales

I Guaales

II Çenboya

III

II Lile

III

I

II La Quebrada

III Lile

III Lile

III Lile

I Guaales

I
Petecui

Xepia

Xepia

Condecoy

Chimba

Cazcoli.

Pontecui

Ponde

Bonba

Bola

Xacoa

Çendo

Yndegua

Bono

Coao

Yali

Piho

Xamundi

Xamundi

Pontecui

Cazcoli

Coao

Bichicama

Xeta

Boo

Xacoa

Petecui

Xeta

Monaco

(Alonso)

Onze

Chiguatoco

Lama

Yndegua

Çendo

Aça

Chiguatoco

Cendo

Bichicama

Condecoy

Xacoa

Xacoa

Chichino

Quincha

Pondo 
649 Combo

650 Come

651 Come

652 Comçacui

653 Cona

654 Cona

655 Conbiche

656 Conco

657 Concui

658 Conchaca

659 Conche

660 Conche

661 Conche

662 Conche

663 Conche

664 Conche

665 Conche

666 Conda

667 Conda

668 Conda

669 Condecoy

670 Condipa

671 Condo

672 Condo

673 Conebo

674 Congo

675 Conilaco

676 Cono

677 Conta

678 Conta

679 Conta

680 Conte

681 Conti

682 Conupa

683 Conçe

684 Coo

III Yande

III Quiguata

III Bitaco/Andra

I La Balsa

III Lile

II La Quebrada

II Bex

III Yande

III Lile

III Lile

III Lile

III Yande/Aqueta

II La Quebrada

III

Monaco

Diacuy

Chiguatoco

Amalile

Amalile

Cazcoli

Sali

Xequino

Yapo

Diacuy

Coao

Coao

Aça

Condecoy

Cayoyo

Çendo

Talima

Chiguatoco

I Piho

II La Quebrada

II Guatonho

III Lile

III

III Yande

I Guaales

II Amayme

III

Piho

Condecoy

Achindeta

Aça

Talima

Diacuy

Monaco

Yambo

Pondo

Xamundi

Xamundi

Xamundi

Lama

Çenboya

Bichicama

I Guaales

Xeta

Petecui

Petecui

Pondo
III

I La Balsa

687 Cota

688 Cota

689 Cota

690 Cotaçi

691 Cotuana

692 Coximba

693 Coy

694 Coy

695 Coy

696 Coya

697 Coyambo

698 Coylo

699 Coylo

700 Coylo

701 Coylo

702 Coylo

703 Coylo

704 Coymbolo

705 Coymbolo

706 Coynda

707 Coyntaça

708 Coynçacoy

709 Coynçacoy

710 Coynçacoy

711 Coynçe

712 Coynçe

713 Coynçecu

714 Coyuca

715 Coyuli

716 Coça

717 Coça

718 Coçe

719 Cuaho

720 Cuano

721 Cuele

722 Cuelo

723 Cueta

724 Cueyo

725 Cui

726 Cuihavache

727 Cuilo

728 Cuilo

729 Cuilo

730 Cuilo

731 Cuilo
III Lile

III Lile

I La Balsa

I

II Ylama

I Guaales

II La Quebrada

II Cuimene

III Lile

III Lile

III Lile

II Aguahax

II Guatonho

I Guaales

I Guaales

III Lile

II La Quebrada

III

III

II La Quebrada

III Lile

II La Quebrada

III Lile

III Lile

II Yohoto

I Guaales

I Guaales

I

III Yande

II Çemboya

III

II Guahande

II La Quebrada

II Yoro

I Guaales

III Yande

II Guahande

II Guahande

III Quiguata
Petecui

Cazcoli

Xacoa

Boo

Cazcoli

Piho

Bonba

Bichicama

Yquinde

(Alonso)

Xequino

Yquinde

Pontecui

Coao

Aça

Coao

Yçando

Achindeta

Chichino

Coao

Condecoy

Petecui

Petecui

Yquinde

Condecoy

Coao

Condecoy

Xacoa

Xacoa

Yqua

(Alonso)

Bichicama

Diacuy

Lama

Talima

Bola

Condecoy

Yho

Emacui

Bola

Bola

Bola

Amalile 
II

I Lile

I Guaales

III

II Çenboya

II Bocota

II La Quebrada

III

II Guatonho

III Bitaco/Baça

II Ylama

II Guahande

II Guatonho

III

II Çenboya

Homa

II Lile

II La Quebrada

II La Quebrada

I Yande

II Yande

III Bitaco/Baça

II Ventayo

II

La Quebrada

I Guaales

Guatonho

III

III Yande

II Bocota

II Capocole

II Ylama

II Ylama

II Yohoto

II La Quebrada

II Çenboya

II Çenboya

II Çenboya
Xacoa

Bichicama

Xeta

Petecui

Lama

Chimba

Bichicama

Condecoy

Petecui

Achindeta

Çendo

Bonba

Bola

Achindeta

Talima

Talima

Lama

Banbayaco

Boo

Xequino

Condecoy

Diacuy

Emacui

Çendo

Chiguatoco

Taqui

Condecoy

Xequino

Piho

(Alonso)

Achindeta

Talima

Xocate

Chimba

Ycobale

Bonba

Bonba

Yqua

Xequino

Lama

Petecui

Lama

$\begin{array}{ll}778 & \text { Chele } \\ 779 & \text { Chembo } \\ 780 & \text { Cheme } \\ 781 & \text { Chempa } \\ 782 & \text { Chempacui } \\ 783 & \text { Chene } \\ 784 & \text { Cheoa } \\ 785 & \text { Chepenoa } \\ 786 & \text { Chí } \\ 787 & \text { Chibi } \\ 788 & \text { Chicaco } \\ 789 & \text { Chico } \\ 790 & \text { Chicoaxe } \\ 791 & \text { Chicobyxa } \\ 792 & \text { Chicocoy }\end{array}$

793 Chicoch

794 Chicotente

795 Chicotone

796 Chicotoni

797 Chicoxemda

798 Chicoximbi

799 Chicoxixe

800 Chicoy

801 Chiche

802 Chiche

803 Chiche

804 Chichino

805 Chichino

806 Chichiro

807 Chiguatoco

808 Chile

809 Chile

810 Chile

811 Chilino

812 Chilo

813 Chilo

814 Chima

815 Chima

816 Chima

817 Chimate

818 Chimba

819 Chimba

820 Chimba

821 Chimbacoy

823 Chimbacui
I Guaales

III Ahoçetala

I

II Tondo

II Capocole

I Guaales

I Guaales

I Guaales

III Ahoçetala

II La Quebrada

II Ventayo

II Yoro

Xeta

Yali

Bichicama

Ajipa

Ycobale

(Alonso)

Xeta

Yali

Condecoy

Onze

Taqui

Chiguatoco

Yho

Pondo

Cayoyo

II Ventayo

Taqui

Cayoyo

Chiguatoco

III

Bono

II Ylama Bonba

Cayoyo

Petecui

Amalile

II Capocole

III Quiguata

III Lile

III

I Guaales

Aça

Bono

Chichino

Yambo

Chiguatoco

II La Quebrada

Coao

III Lile

III Lile

III Ahoçetala

I Guaales

II Capocole

III Lile

III Lile

III Lile

III

II Bocota

II Guatonho

II Ylama

II La Quebrada

II La Quebrada
Coao
Yali

Chichino

Ycobale

Xacoa

Xacoa

Xacoa

Talima

Chimba

Achindeta

Bonba

Onze

Condecoy

Xequino 
824 Chimbo

825 Chimbo

826 Chimboa

827 Chimbonbo

828 Chime

829 Chime

830 Chimpa

832 Chindacu

833 Chindian

834 Chintiqua

835 Chiqui

836 Chita

837 Chita

838 Chitacoy

839 Chitacui

840 Cho.oque

841 Choa

842 Choasi

843 Choca

844 Chocoz

845 Chocunbache

846 Chocho

847 Chogata

848 Choguache

849 Choguacho

850 Choho

851 Choho

852 Chole

853 Cholo

854 Cholo

855 Choma

856 Chomba

857 Chomba

858 Chomba

859 Chombe

860 Chomecui

861 Chomende

862 Chonche

863 Choncho

864 Chonde

865 Chone

866 Chone

867 Chone

868 Chone

\section{III}

II Guatonho

Petecui

II La Quebrada

II Quiguez

III Yande

II Cuimene

III Lile

II Ylama

II Amayme

II Homa

III Lile

III Lile

III

II La Quebrada III

II Homa

III Lile

II Homa

II Ventayo

II Ylama

III Lile

II Ylama

II Ylama

II Ylama

III

III

II Yoro

III Lile

I

III Bitaco/Andra

III Yande

III Yande

II Cuimene

II Cenboya

III Quiguata

II Guahande

III Lile

II Cenboya

II Homa

II Cenboya

II Cenboya

II Cenboya

II Guatonho

III Lile
Achindeta

Cendo

Condecoy

Guanche

Emacui

Pontecui

Xacoa

Bonba

Yambo

Banbayaco

Sali

Coao

Petecui

Condecoy

Talima

Bambayaco

Aca

Banbayaco

Taqui

Bonba

Sali

Bonba

Bonba

Bonba

Petecui

Petecu

Yho

Coao

Bichicama

Emacui

Diacuy

Pontecui

Lama

Amalile

Bola

Xacoa

Banbayaco

Lama

Lama

Lama

Archindeta

Boo
870 Chonibamba

871 Chonsi

872 Chonta

873 Chonta

874 Choo

875 Choti

876 Chu

877 Chuche

878 Chuguadix

879 Chuguela

880 Chuguex

881 Chuha

882 Chuhuela

883 Chui

884 Chula

885 Chuli

886 Chumba

887 Chumba

888 Chumbo

889 Chumbo

890 Chumbocui

891 Chume

892 Chumende

893 Chumpaca

894 Chumpidina

895 Chupa

896 Chupe

897 Dacacui

898 Dacui

899 Dachancui

900 Dagua

901 Dagua

902 Dagua

903 Dagua

904 Daguaya

905 Dague

906 Dalima

907 Dame

908 Danda

909 Danda

910 Dandacoy

911 Dandacui

912 Dandacui

913 Dandacui

914 Dandacui
II Guahande

III Bitaco/Baça

II Guahande

II La Quebrada

III

III Lile

II Ylama

II Ylama

II Guatonho

II Lile

II Ylama

II Lile

II Ylama

II Yohoto

I Bex

III Ahoçetala

II Bitaco/Andra

I Yohoto

I La Quebrada

II Guimene

II Ylama

II Guatonho

III Yande

III Yande/Aqueta

II Çenboya

II Yohoto

II Bitaco/Baça

II Ylama

II Ylama

II Ylama

III

I La Balsa

II Homa

III Lile

III

II Bex

II Tondo

III

III
Bola

Bola

Condecoy

Petecui

Xita

Bonba

Bonba

Achindeta

Xacoa

Bonba

Sali

Bonba

Yqua

Chumba

Yapo

Yali

Talima

Yqua

Condecoy

Pontecui

Bichicama

Bonba

Achindeta

Diacuy

Lama

Yqua

Bonba

Bonba

Bonba

Petecui

Cazcoli

Banbayaco

Aça

Talima

Xita

Yapo

Ajipa

Talima

Talima 
915. Dandacui

916 Dandacui

917 Dandacui

918 Dandacui

919 Dapa

920 Dapa

921 Dapa

922 Dapa

923 Dapa

924 Dapa

925 Dapano

926 Dape

927 Dape

928 Dape

929 Dape

930 Dape

931 Dape

932 Dape

933 Dapa

934 Datoma

935 Dax

936 Decala

937 Decoy

938 Decui

939 Degua

940 Degua

941 Dehe

942 Dehecoy

943 Demacaçi

944 Demda

945 Demo

946 Depe

947 Dequi

948 Deto

949 Deuma

950 Dex

951 Deçe

952 Deçe

953 Deçe

954 Dia

955 Dia

956 Dia

957 Diacui

958 Dian

959 Diary

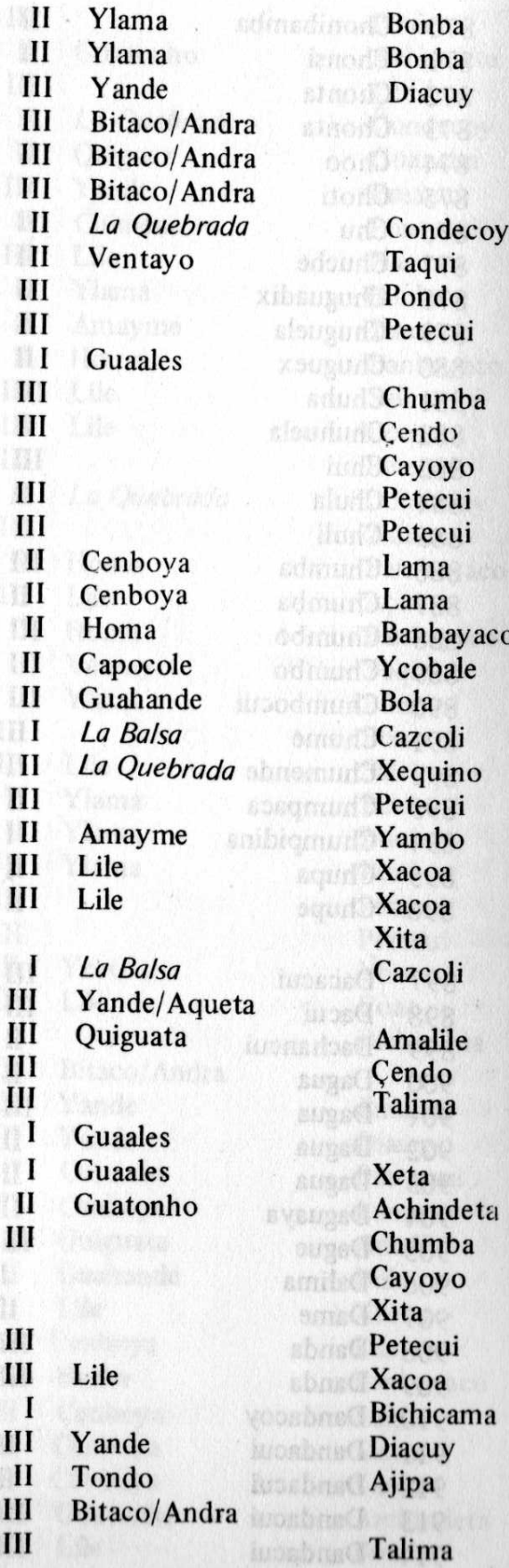

I La Balsa

962 Dico

963 Dico

964 Dico

965 Dicoiz

966 Dicui

967 Dicui

968 Dicui

969 Dicui

970 Dicui

971 Dieta

972 Digua

973 Dilama

974 Dilichi

975 Dima

976 Dima

977 Dimagua

978 Dimixel

979 Dimixela

980 Dimo

981 Dinde

982 Dinde

983 Dinde

984 Dinde

985 Dinde

986 Dindi

987 Dino

988 Dipa

989 Dipe

990 Diquiyoa

991 Disqui

992 Dita

993 Ditecui

994 Diti

995 Diu egale

996 Dix

997 Dix

998 Dixe

999 Dociyaua

1.000 Dola

1.001 Donda

1.002 Donda

1.003 Donde

1.004 Dondo

1.005 Dondo

1.006 Dondo
I Guaales

Cazcoli

Xeta

Bichicama

Petecui

Bono

Benbacui

Ypiçe

Achindeta

Yho

Amalile

Amalile

Talima

Xeta

Coao

Ajipa

Petecui

Xepia

Monaco

Chumba

Amalile

Amalile

Ycobale

Achindeta

Pontecui

Achindeta

Petecui

Petecui

Bichicama

Bitaco/Baca

II Ahoçetala

II Capocole

II Ventayo

Talima

Yali

Ycobale

Taqui

Cayoyo

Bono

Bonba

Pondo

(Alonso)

(Alonso)

Xocate

Achindeta

Bonba

Bichicama

Yapo

Piho 
1.007 Dondo

1.008 Dondo

1.009 Dondo

1.010 Dondo

1.011 Dondo

1.012 Dondo

1.013 Dondo

1.014 Dooa

1.015 Doxe

1.016 Doxe

1.017 Doyna

1.018 Doyna

1.019 Doyna

1.020 Doyne

1.021 Doyne

1.022 Doyne

1.023 Doçe

1.024 Doçe

1.025 Doze

1.026 Ducache

1.027 Dunda

1.028 Dundo

1.029 Duuçe

1.030 Duçe

1.031 Eaxunu

1.032 Eca

1.033 Ecaca

1.034 Echecul

1.035 Econa

1.036 Echecaca

1.037 Echecoy

1.038 Echecui

1.039 Echecui

1.040 Echila

1.041 Echimbo

1.042 Echiqui

1.043 Egue

1.044 Egue

1.045 Egui

1.046 Ehe

1.047 Emacui

1.048 Emba

1.049 Emba

1.050 Embacui

1.051 Embaxis

I) I

$\begin{aligned} \text { I Guaales } & \text { Ponde }\end{aligned}$

Lile Aça

Petecui

Petecui

Yoro Bon

III Chumba

III La Quebrada $\quad \begin{array}{ll}\text { Petecui } \\ \text { Condecoy }\end{array}$

II Lile Xacoa

II Guaales (Alonso)

Petecui

Petecui

Petecui

III

I Guaales

III Lile

I Guaales

III Lile

III

III Lile

III Lile

III Lile

III Yande

III Lile

II La Quebrada

I

Condecoy

II Yande Iquinde

Petecui

III Lile Aça

III Yande Quindecui

III

II Guatonho

II La Quebrada

Chumba

Achindeta

I Guaales

III Yande

II La Quebrada

II Aguahax

III Yande

II Guatonho
1.052 Embaxis

1.053 Embe

1.054 Embecui

1.055 Embi

1.056 Embo

1.057 Embo

1.058 Embo

1.059 Embox is

1.060 Emxa

1.061 Enche

1.062 Endagua

1.063 Endo

1.064 Ene

1.065 Ene

1.066 Eno

1.067 Eno

1.068 Eno

1.069 Ente

1.070 Encemba

1.071 Encembo

1.072 Ençuqui

1.073 Eoquicho

1.074 Epalo

1.075 Epas

1.076 Epique

1.077 Eti

1.078 Eto

1.079 Euna

1.080 Ex

1.081 Exaha

1.082 Exaha

1.083 Execonche

1.084 Execonche

1.085 Execui

1.086 Execui

1.087 Execui

1.088 Exede

1.089 Exelee

1.090 Exembo

1.091 Exeoçe

1.092 Exeta

1.093 Exetahe

1.094 Exetecui

1.095 Exetoco

1.096 Exetoco

1.097 Exetoco
II Guatonho

III Lile

III

III Bitaco/Andra

III

III Lile

II Guatonho

III Ahocetala

III

III Bitaco/Baça

II Yoro

III Lile

III

II La Quebrada

I Guaales

III

II Çenboya

III Bitaco/Andra

II Yohoto

II Yohoto

I Guaales

\section{III}

III

II La Quebrada

II Ventayo

I Guaales

I Guaales

II Bocota

III

II Çenboya

II Cuimene

III Yande

II Bex

II Bocota

II Bocota

II Guahande

II Guahande

II Guahande

II Yoro

II Guatonho
Achindeta

Sali

Talima

Talima

Xacoa

Achindeta

Yali

Yndegua

Talima

Yho

Aca

Onze

Petecui

Xequino

Monaco

Çendo

Lama

Talima

Yqua

Yqua

Chiguatoco

Petecui

Talima

Xequino

Taqui

Ponde

Ponde

Chimba

Yndegua

Petecui

Lama

Pontecui

Cayoyo

Quindecui

Yapo

Chimba

Chimba

Bola

Bola

Bola

Yho

Achindeta 07 
II Çenboya

II Capocole

II Guahande

II Yoro

II Guahande

III Bitaco/Baça

1.104 Exipa

1.105 Eyuncui

1.106 Eyunqui

1.107 Eçaha

1.108 Eçe

1.109 Eçecambo

1.110 Eçepe

1.111 Gahocho

1.112 Gambaa

1.113 Gamboçe

1.114 Ganchi

1.115 Gate

1.116 Gate

1.117 Gate

1.118 Gavitia

1.119 Gazaca

1.120 Gemdo

1.121 Goama

1.122 Goana

1.123 Goano

1.124 Goeyoçe

1.125 Goguembo

1.126 Goncagui

1.127 Goo

1.128 Goxingoa

1.129 Goxingoca

1.130 Guaame

1.131 Guaaya

1.132 Guacaca

1.133 Guacamita

1.134 Guacanoa

1.135 Guacata

1.136 Guaçequi

1.137 Guacui

1.138 Guacha

1.139 Guache

1.140 Guachinde

1.141 Guagu

I Bocota

II Guahande

II La Quebrada

I La Balsa

III

Lama
Ycobale
Emacui
Bola
Yho
Bola
Cayoyo
Cayoyo
Xeta
Petecui
Bola
Chimba

Bola
Xequino
Cazcoli
Pondo
Onze
Petecui
Xacoa
Cazcoli
Sali
Piho
Petecui
Petecui
Xita
Xamundi
Talima
Talima
Monaco
Cazcoli
Xamundi
Ponde
Yali
Onze
Xamundi
Xeta
Chumba
Chumba
Yapo
Talima
Bola
Yquinde
Petecui
Pontecui

1.143 Guaguacoy

1.144 Guaguacoy

1.145 Guaguacuy

1.146 Guagueco

1.147 Guahambo

1.148 Guahana

1.149 Guahapo

1.150 Guahole

1.151 Gual

1.152 Guala

1.153 Guali

1.154 Guamache

II Guahande

1.155 Guamache

1.156 Guamba

1.158 Guambia

1.159 Guambia

1.160 Guana

1.161 Guana

1.162 Guanache

1.163 Guanavo

1.164 Guanaxo

1.165 Guanaxo

1.166 (Guancavelico)

1.167 Guançecoy

1.168 Guanche

1.169 Guanchecoy

1.170 Guandi

1.171 Guandica

1.172 Guane

1.173 Guanto

1.174 Guançecoy

1.175 Guapehende

1.176 Guapendo

1.177 Guapendo

1.178 Guapendo

1.179 Guaqui

1.180 Guaquich

1.181 Guaquiche

1.182 Guaquichecui

1.183 Guatacui

1.184 Guatamache

1.185 Guatax

1.186 Guate

1.187 Guatima

1.188 Guatinchinde
La Quebrada

Condecoy

Bono

Petecui

Talima

Chiguatoco

Xeta

Cayoyo

III Bitaco/Andra

I Guaales

II Ventayo

II Cenboya

I Guaales

Taqui

Lama

Xeta

Petecui

Yquinde

Achindeta

Coao

Xamundi

Yndegua

Cayoyo

Chichino

Guaales

II Yoro

III Lile

III

II Quiguez

II Guatonho

III Yande

I La Balsa

Aça

Talima

Yndegua

Guanche

Achindeta

Quindecui

Petecui

Cazcoli

Petecui

Talima

Cayoyo

Xequino

Petecui

Bono

Talima

Bonba

Yho

Pontecui

Ycobale

Piho

Lama

Petecui

Cazcoli

Lama 


$\begin{array}{ll}1.189 & \text { Guaxa } \\ 1.190 & \text { Guaxa } \\ 1.191 & \text { Guaxaxos } \\ 1.192 & \text { Guaxeno } \\ 1.193 & \text { Guaxotauma } \\ 1.194 & \text { Guaxume } \\ 1.195 & \text { Guaya } \\ 1.196 & \text { Guaya } \\ 1.197 & \text { Guaya } \\ 1.198 & \text { Guaya } \\ 1.199 & \text { Guayama } \\ 1.200 & \text { Guayo } \\ 1.201 & \text { Guayo } \\ 1.202 & \text { Guayo } \\ 1.203 & \text { Guayos } \\ 1.204 & \text { Guayoçe } \\ 1.205 & \text { Guayoz } \\ 1.206 & \text { Guayoz } \\ 1.207 & \text { Guayuz } \\ 1.208 & \text { Guayuz } \\ 1.209 & \text { Gucande } \\ 1.210 & \text { Guecos } \\ 1.211 & \text { Guecho } \\ 1.212 & \text { Gueha } \\ 1.213 & \text { Guehe } \\ 1.214 & \text { Guembo } \\ 1.215 & \text { Guemo } \\ 1.216 & \text { Guenbo } \\ 1.217 & \text { Guenbo } \\ 1.218 & \text { Guenca } \\ 1.219 & \text { Guencha } \\ 1.220 & \text { Guenchecoy } \\ 1.221 & \text { Guenchecui } \\ 1.222 & \text { Guendo } \\ 1.223 & \text { Gueneçe } \\ 1.224 & \text { Guenic } \\ 1.225 & \text { Guençacoy } \\ 1.226 & \text { Guençecoy } \\ 1.227 & \text { Guençecoy } \\ 1.228 & \text { Guencecoy } \\ 1.229 & \text { Guençecoy } \\ 1.230 & \text { Guençecoy } \\ 1.231 & \text { Guençecoy } \\ 1.232 & \text { Gueriz } \\ 1.233 & \text { Guete } \\ 1.234 & \text { Guetecoy } \\ & \end{array}$

\begin{tabular}{r|l} 
II & Bocota \\
III & \\
III & \\
III & \\
I & \\
I & Guaales \\
I & Guaales \\
I & Guaales \\
& \\
II & Guahande \\
III & \\
III & \\
III & \\
III & \\
III & \\
II & Guatonho \\
II & Çenboya \\
II & Guahande \\
III & Lile \\
I & \\
I & \\
III & \\
& \\
II & Homa \\
III & Lile \\
III & \\
\hline
\end{tabular}

Chimba

Cendo

Pondo

Pondo

Xamundi

Chichino

Xeta

Ponde

Cayoyo

Bola

Chumba

Petecui

Petecui

Talima

Talima

Achindeta

Lama

Bola

Coao

Bichicama

Bichicama

Talima

Cayoyo

Banbayaco

Aça

Çendo

Chiguatoco

Cayoyo

Ajipa

Bonba

Aça

II Ylama

III Lile

II Guatonho

II La Quebrada

Chiguatoco Achindeta

Xequino

Pondo

Coao

Condecoy

Condecoy

Petecui

Çendo

Cendo

Bichicama

Yquinde

Emacui

Chiguatoco

Pondo

$\begin{array}{ll}1.235 & \text { Guex } \\ 1.236 & \text { Guex } \\ 1.237 & \text { Guexe } \\ 1.238 & \text { Guexo } \\ 1.239 & \text { Gueyco } \\ 1.240 & \text { Gueyoca } \\ 1.241 & \text { Gueça } \\ 1.242 & \text { Guez } \\ 1.243 & \text { Guez } \\ 1.244 & \text { Guez } \\ 1.245 & \text { Guicha } \\ 1.246 & \text { Guicha } \\ 1.247 & \text { Guinxe } \\ 1.248 & \text { Guinxe } \\ 1.249 & \text { Guinxi } \\ 1.250 & \text { Guinça } \\ 1.251 & \text { Guinça } \\ 1.252 & \text { Guinçacoy } \\ 1.253 & \text { Guinçacui } \\ 1.254 & \text { Guinçaquiy } \\ 1.255 & \text { Guinçe } \\ 1.256 & \text { Guinçe } \\ 1.257 & \text { Guite } \\ 1.258 & \text { Guiçucui } \\ 1.259 & \text { Guyuz } \\ & \\ 1.260 & \text { Hacte } \\ 1.261 & \text { Hacua } \\ 1.262 & \text { Hagoa } \\ 1.263 & \text { Hahache } \\ 1.264 & \text { Hambo } \\ 1.265 & \text { Hambo } \\ 1.266 & \text { Hambro } \\ 1.267 & \text { Hampaxe } \\ 1.268 & \text { Hampo } \\ 1.269 & \text { Han } \\ 1.270 & \text { Hande } \\ 1.271 & \text { Hanqui } \\ 1.272 & \text { Hanqui } \\ 1.273 & \text { Haxcui } \\ 1.274 & \text { Hechacui } \\ 1.275 & \text { Heche } \\ 1.276 & \text { Hechecui } \\ 1.277 & \text { Hecho } \\ 1.278 & \text { Hemba } \\ 1.279 & \text { Hemhe }\end{array}$

Coao

Coao

Talima

Talima

Taqui

Ajipa

Pondo

Çendo

Petecui

Diacuy

Petecui

Condecoy

Banbayaco

Bichicama

Petecui

Cazcoli

La Balsa

Guatonho

Guatohno

chindeta

Petecui

Achindeta

Talima

Xita

Petecui

Lile

Coao

Talima

Chumba

II Yande

I La Quebrada

Diacuy

Bichicama

Condecoy

Bonba

Quindecui

III Yande

II Guatonho

Achindeta

Chumba

Chumba

Achindeta

Guatonho

II Ventayo

III Ahoçetala

Taqui

Yali

III Bitaco/Baça

Xita

Cayoyo

Achindeta

II Guatonho

Petecui

II Yohoto

Guaales

I Aguahax

Yqua

(Alonso)

Yçando

Talima 
1.280 Hicamba

1.281 Hicanboz

1.282 Hicgue

1.283 Hicguec

1.284 Hicpaiac

1.285 Hicto

1.286 Hidoc

1.287 Hindapa

1.288 Hindecui

1.289 Hinuzembo

1.290 Hipoyo

1.291 Hiquetonça

1.292 Hiquipa

1.293 Hiyque

1.294 Hoapa

1.295 Hocoy

1.296 Homo

1.297 Honqui

1.298 Honçe

1.299 Hopiha

1.300 Hotacui

1.301 Hoxtena

$1.302 \mathrm{Hu}$

1.303 Hueçecoy

1.304 Hueco

1.305 Huelahache

1.306 Huequa

1.307 Huete

1.308 Huete

1.309 Huetecui

1.310 Gueço

1.311 Huita

1.312 Huiticoe

1.313 Hunba

1.314 Hunca

1.315 Huton

1.316 Huyqui

II Bex

II Guatonho

II Ylama

II Guatonho

II Ylama

II Quiguez

III Dagua

III Bitaca/Baça

III Quiguata

III Quiguata

III Bitaco/Baça

II Hom

III Lile

II La Quebrada

II Bocota

III

II Guatonho

II Guatonho

III

III Iil

Lile Aça

Bola

I Guahande

II Guahande

III Ahoçetala

II Cuimene

III Yande

III Yande

III Bitaco/Andra

III Lile

II Guatonho

II Cuimene

III Yande

I Guaales

II La Quebrada

II Ylama

II Guatonho
Cayoyo

Yapo

Achindeta

Bonba

Achindeta

Bonba

Xita

Guanche

Benbacui

Amalile

Amalile

Banbayaco

Coao

Condecoy

Chimba

Achindeta

Achindeta

Chumba

onba

Bonba
Bola
Talima

Talima

Bola

Cayoyo

Yali

Pontecui

Emacui

Quindecui

Xacoa

Achindeta

Pontecui

Emacui

(Alonso)

Condecoy

Bonba

Achindeta
1.324 Inme

1.325 Inpehex

1.326 Ixiquinbi

1.327 Ioco

1.328 Lac

$1.329 \mathrm{Lac}$

1.330 Laca

1.331 Laca

1.332 Lagua

1.333 Laha

1.334 Lahac

1.335 Lala

1.336 Lala

1.337 Lala

1.338 Lala

1.339 Lalo

1.340 Lama

1.341 Lama

1.342 Lama

1.343 Lama

1.344 Lama

1.345 Lama

1.346 Lama

1.347 Lama

1.348 Lama

1.349 Lamachico

1.350 Lamascache

1.351 Lamba

1.352 Lambi

1.353 Lanucoy

1.354 Laoy

1.355 Laoy

1.356 Lape

1.357 Laqua

1.358 Laque

1.359 Laqui

1.360 Lati

1.361 Laxo

1.362 Layaqui

1.363 Laçe

1.364 Lembo

1.365 Lembo

1.366 Leme

1.367 Leme
II Guahande

Ylama

II La Quebrada

III

Bonba

Xequino

Talima

Quindecui

Diacuy

Aça

Xacoa

Amalile

Petecui

Pondo

Petecui

Petecui

Talima

(Alonso)

Pondo

Chiguatoco

Bonba

Diacuy

Amalile

Chimba

Lama

Aça

Aça

Laca

Xeta

Ponde

Bonba

Lama

Pondo

Pondo

Petecui

Boo

Bonba

Yambo

Taqui

Xequino

Xepia

Talima

Xamundi

Banbayaco

Achindeta

Xequino

Yquinde 
1.368 Lengacoy

1.369 Leça

1.370 Lichi

1.371 Lile

1.372 Lile

1.373 Lile

1.374 Lile

1.375 Lile

1.376 Lile

1.377 Lile

1.378 Lile

1.379 Lile

1.380 Lile

1.381 Lile

1.382 Lile

1.383 Limba

1.384 Lisqua

1.385 Loa

1.386 Loa

1.387 Loaza

$\begin{array}{ll}1.388 & \text { Loco } \\ 1.389 & \end{array}$

1.390 Locoeto

1.391 Loche

1.392 Lolo

1.393 Loma

1.394 Lombe

1.395 Lombo

1.396 Lomi

1.397 Loque

1.398 Loque

1.399 Loyno

1.400 Luca

1.401 Luchica

1.402 Luma

1.403 Lumba

1.404 Lumba

1.405 Lumbe

1.406 Lumbi

1.407 Lumbi

1.408 Lumbi

1.409 Lumbi

1.410 Lume

1.411 Lume

1.412 Lume
III Lile

III Lile

II Guatonho

III Lile

III Ahoçetala

III

III Dagua

III Dagua

III Bitaco/Andra

II Bitaco/Baça

II Ylama

II Ylama

II Ylama

I Guaales

II Guahande

III

II La Quebrada

III Yande

III

II La Quebrada

III

II La Quebrada

II Guatonho

I Guaales

III

III

III Lile

III Lile

III

III Lile

I Guaales

I Guaales

II Yohoto

II La Quebrada

II Guahande

II Çenboya

III Lile

III Lile

I Guaales

III Bitaco/Andra

II Bitaco/Baça
Xita

Boo

1.414 Mac çana

Achindeta

Boo

Yali

Talima

Ypiçe

Benbacui

Bonba

Bonba

Bonba

Xeta

Bola

Chumba

Xequino

Emacui

Petecui

Pondo

Xequino

Bono

Xequino

Achindeta

Xeta

Talima

Bichicama

Bono

Aça
Aça
Pond

Pondo

Sali

Monaco

Xeta

Yqua

Xequino

Bola

Lama

Aça

Aça

Monaco

Bichicama

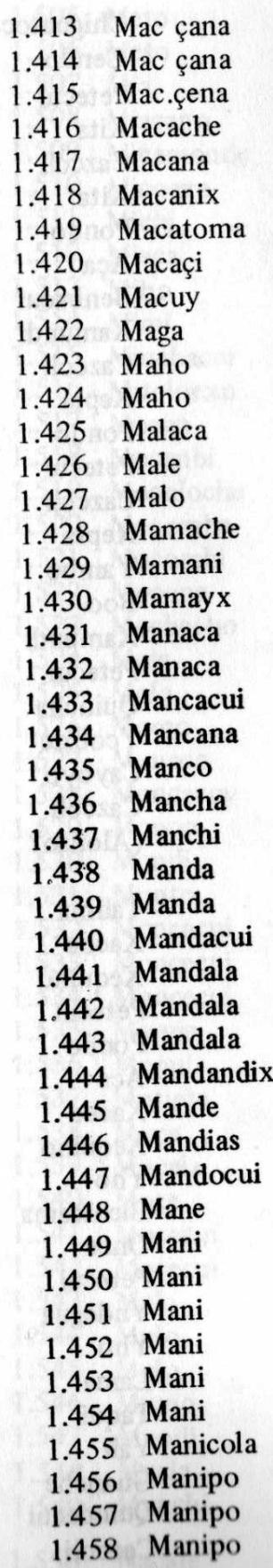

I

I

Lile

I La Balsa

I La Balsa

III Lile

I Guaales

III

III

I Guaales

II Homa

II Amayme

III

III Homa

III Lile

III Lile

II Aguahax

III Lile

II Yoro

III

II Yoro

II Homa

I Guaales

III Yande

III Ahoçetala

II Yoro

II Amayme

II Guahande

III

III

III

III

III

II Guahande

I

II

Ventayo

Xamundi

Xamundi

Xamundi

Xepia

Sali

Piho

Cazcoli

Cazcoli

Sali

Monaco

Ponde

Petecui

Petecuy

Xeta

Banbayaco

Petecui

Yambo

Indegua

Petecui

Petecui

Banbayaco

Sali

Coao

Yçando

Xacoa

Yho

Petecui

Yho

Banbayaco

Xeta

Diacuy

Yali

Yho

Yambo

Bola

Talima

Petecui

Petecui

Petecui

Petecui

Petecui

Bola

Monaco

Quincha

Taqui

Petecui 
Chiguatoco

1.461 Manocoy

1.462 Manta

1.463 Manticala

1.464 Manto

1.465 Manto

1.466 Manto

1.467 Mançecui

1.468 Mapinoa

1.469 Maponga

1.470 Maqui

1.471 Maqui

1.472 Maqui

1.473 Mascala

1.474 Mascapa

1.475 Matati

1.476 Mate

1.477 Maxela

1.478 Maxila

1.479 Maya

1.480 Mayo

1.481 Maço

1.482 Mazcapa

1.483 Meacanoa

1.484 Mecata

1.485 Meche

1.486 Meche

1.487 Melaqu

1.488 Melo

1.489 Meme

1.490 Meme

1.491 Meme

1.492 Meme

1.493 Memetoho

1.494 Memçe

1.495 Menca

1.496 Menche

1.497 Mença

1.498 Mençe

1.499 Mençe

1.500 Menz

1.501 Menza

1.502 Meo

1.503 Mequi

1.504 Meta

Petecui

Xita

I La Balsa

Cazcoli

Xita

III Lile Pondo

III Dagua

La Balsa

Aça

Benbacui

Xamundi

Cazcoli

Xepia

Pondo

Petecui

La Balsa

Cazcoli

Xepia

Yambo

Boo

Xamundi

Petecui

Quincha

I Capocoli

Ycobale

Cayoyo

I La Balsa

I Guaales

III

III Lile

I La Quebrada

Cazcoli

(Alonso)

Talima

Xacoa

Xequino

Petecui

III Pondo

III Lile Aça

III Lile Xacoa

II La Quebrada Xequino

I Yoro

Yho

Bichicama

III Onze

III Petecui

II Yoro $\quad$ Yho

II Çenboya Lama

II Ventayo

III Ahoçetala

Taqui

II Quiguez $\quad$ Guanch

III Yande Quindecui

I LaBalsa Cazcoli
1.505 Meto
1.506 Meto

I Guaales
$1.507 \mathrm{Mia}$

1.508 Miacano

1.509 Micamonde

1.510 Micoaxe

1.511 Michi

1.512 Mieca

1.513 Miho

1.514 Mimi

1.515 Minchacui

1.516 Mitoloexo

1.517 Moago

1.518 Mocanbi

1.519 Mocoloche

1.520 Mocombe

1.521 Mocomb

1.522 Mocone

1.523 Mochecho

1.524 Mola

1.525 Mola

1.526 Momo

1.527 Monaco

1.528 Moncacoy

1.529 Monco

1.530 Mondi

1.531 Monto

1.532 Monçacui

1.533 Monçecui

1.534 Monçecui

1.535 Moque

1.536 Moqui

1.537 Motiet

1.538 Moça

1.539 Moçala

1.540 Moçe

1.541 Moçecui

1.542 Moçecui

1.543 Mula

1.544 Mulo

1.545 Mulo

1.546 Mumo

1.547 Mundi

1.548 Myala

1.549 Mynchi

1.550 Nacate
Bichicama

Petecui

Cendo

(Alonso)

Monaco

Xamundi

Condecoy

Yho

(Alonso)

Lama

Bonba

Xamundi

Yndegua

Petecui

(Alonso)

Chimba

Xeta

Cazcoli

Yho

Ypiçe

Benbacui

Condecoy

Monaco

Bichicama

Talima

I

III

III Quiguata

II La Quebrada

Amalile

I Yoro

II Bocota

II La Quebrada

II Ylama

II Yande/Aqueta

I La Balsa

Condecoy

Chumba

Yho

Chimba

Yndegua

Condecoy

III Bitaco/Andra

II Guahande

I Guaales

Bonba

Cazcoli

Petecui

II Ventayo

Bola

Monaco

Taqui

Chiguatoco

Cayoyo

I Guaales

Xeta

Pondo

III Lile Aça

III 
$1.551 \quad \mathrm{Naco}$

1.552 Nacono

1.553 Namaçe

1.554 Namba

1.555 Nambacui

1.556 Nambacui

1.557 Name

1.558 Namhe

1.559 Nana

1.560 Nanxo

1.561 Nasche

1.562 Neca

1.563 Negua

1.564 Nema

1.565 Nenca

1.566 Nenca

1.567 Nencacoy

1.568 Nencacoy

1.569 Nencacu

1.570 Nencacui

1.571 Nenga

1.572 Nengacoy

1.573 Nengacui

1.574 Nengacui

1.575 Nenpoa

1.576 Nepo

1.577 Nesxe

1.578 Niaca

1.579 Niache

1.580 Nianquapelo

1.581 (Nicaragua)

1.582 Nilax

1.583 Ninecui

1.584 Niunqua

1.585 Nizimba

1.586 No

1.587 Noa

1.588 Noa

1.589 Noa

1.590 Noadaxe

1.591 Noan

1.592 Noaxele

1.593 Nochoque

1.594 Noe

1.595 Nomcho

1.596 Nooa
II Yoro

I Guaales

Yho

(Alonso)

Xamundi

III Bitaco/Andra

III Yande

III Bitaco/Baça

III Ahoçetala

I Guaales

I

II Ylama

III Lile

I

III Lile

II Yoro

III

II Lile

III Bitaco/Andra

II Yoro

III

II Guahande

III Bitaco/Andra

Ylama

Guatonho

Ylama

Ylama

Bitaco/Andra

I Homa

II Ventayo

III

I

III

II Amayme
Cendo

Diacuy

Yali

Xeta

Xamundi

Bonba

Boo

Quincha

Aça

Petecui

Yho

Bono

Xacoa

Cayoyo

Yho

Pondo

Bola

Xamundi

Xamundi

Bonba

Petecui

Achindeta

Bonba

Petecui

Bonba

Banbayaco

Taqui

Bichicama

Bichicama

Talima

Cendo

Yambo

Xamundi

Petecui

Yquinde

Talima

Xepia
1.597 Notana

$1.598 \mathrm{Nu}$

1.599 Nylo

1.600 Name

$1.601 \mathrm{Oa}$

1.602 Oabis

1.603 Oala

1.604 Oama

1.605 Oambo

1.606 Oan

1.607 Oandacui

1.608 Oaño

1.609 Oaoy

1.610 Oaqui

1.611 Oaquiche

1.612 Oaquiz

1.613 Oate

1.614 Oau.y

1.615 Oau.ym

1.616 Oaçe

1.617 Oazacui

1.618 Ocache

1.619 Ocando

1.620 Ocapando

1.621 Oco

1.622 Oco

1.623 Ocomaye

1.624 Ocomo

1.625 Ocuchacui

1.626 Ocui

1.627 Ocui

1.628 Ocumbo

1.629 Ocuybo

1.630 Ochoho

1.631 Oechendechi

1.632 Oemacui

1.633 Oembi

1.634 Oembo

1.635 Oemecui

1.636 Oemecui

1.637 Oemecui

1.638 Oendo

1.639 Oenx

1.640 Oenxecui

1.641 Oepa
I Guaales

III Lile

III Lile

III Ahoçetala

Xacoa

Boo

Yali

II Yoro

II Bocota

II Guahande

III

I Quiguata

III Bitaco/Baça

III Quiguata

II Cenboya

II La Ouebrada

II Cenboy

III Lile

II La Quebrada

II Yoro

II Guahande

II Çenboya

III Ahoçetala

II Homa

III Bitaco/Baça

III

III

III

III

III Yande

II Bocota

III Lile

III Lile

II Yoro

II Bocota

II Capocole

II Cenboya

II Cenboya

II Cenboya

III Quiguata

II Cenboya

II Capocole

III Bitaco/Andra

I Guaales

\section{Yho}

Chimba

Bola

Petecui

Quincha

Amalile

Amalile

Lama

Xequino

Lama

Coao

Condecoy

Yho

Bola

Lama

Yali

Quincha

Banbayaco

Petecui

Bono

Petecui

Piho

Petecui

Xocate

Chimba

Coao

Aça

Yho

Chimba

Ycobale

Lama

Bono

Lama

Lama

Amalile

Lama

Ycobale 
1.643 Oexa

1.644 Oexe

1.645 Oexo

1.646 Oeçecui

1.647 Ogochiche

1.648 Ohecax

1.649 Ohecoy

1.650 Ohequi

1.652 Oinche

1.653 Oli

1.654 Olima

1.655 Olis

1.656 Olla

1.657 Oloy

1.658 Oloce

1.659 Oltecui

1.660 Oma

1.661 Omachimbo

1.662 Omanda

1.663 Omba

1.664 Ombacoy

1.665 Ombacoy

1.666 Ombocui

1.667 Omboye

1.668 Omexe

1.669 Omolile

1.670 Ompi

1.671 Onde

1.672 Onde

1.673 Onde

1.674 Ondi

1.675 Ongu.e

1.676 Onze

1.677 Ooaca

1.678 Ooçe

1.679 Opama

1.680 Opama

1.681 Opolo

1.682 Oqui

1.683 Oqui

1.684 Oqui

1.685 Oretoco

1.686 Otaco
I La Balsa

I La Balsa

Cazcoli

Cazcoli

Chimba

Ponde

I Guaales

II Cenboya

II Aguahax

III Lile

III Yande

III

III Lile

II Capocole

III Lile

III

I Guaales

II Cenboya

III Lile

III Quiguata

III Quiguata

III Yande

III Lile

III Lile

III Bitaco/Baça

II La Quebrada

II Yoro

III Quiguata

III Quiguata

III

III

II Yoro

II La Quebrada

III

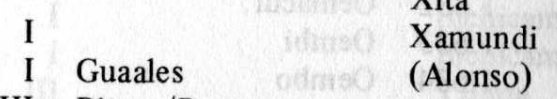

III Bitaco/Baça

III

III

II Bocota

II Guahande

Pondo
Cendo

Bola

III Dagua

Yçando

Coao

Xita

Quindecui

Xita

Pond

Poo
Petecui

Ycobale

Boo

Pondo

Lama

Sali

Amalile

Amalile

Xoca

Aça

Xequino

Yho

Amalile

Amalile

Lama

Petecui

Chumba

Yho

Condecoy

Onze

Chumba

(Alonso)

ondo

Chimba

Benbacui
Bonba

III Bitaco/Baça

III Bitaco/Baça

II Cuimene
1.689 Otapo

1.690 Otapo

1.691 Otapo

1.692 Otapo

1.693 Otata

1.694 Otencu

1.695 Otencui

1.696 Otindica

1.697 Otmecui

1.698 Oto

1.699 Oto

1.700 Oto

1.701 Otondica

1.702 Otondica

1.703 Otumba

1.704 Otumbe

$1.705 \mathrm{Ou}$

1.706 Oxande

1.707 Oxende

1.708 Oxoma

1.709 Oya

1.710 Oyco

1.711 Oycui

1.712 Oyga

1.713 Oyla

1.714 Oyla

1.715 Oylambo

1.716 Oyme

1.717 Oyme

1.718 Oynacui

1.719 Oynce

1.720 Oynche

1.721 Oynde

1.722 Oynga

1.723 Oyniche

1.724 Oynçacoy

1.725 Oyocui

1.726 Oyondo

1.727 Oysqui

1.728 Oyte

1.729 Oyte

1.730 Oyticoy

1.731 Oyumçique

1.732 Oyundocoy

1.733 Oça
II Guatonho

II Yohoto

III Bitaco/Baça

II Çenboya

I Guaales

III

III Lile

II Guahande

III

III

Lile

La Balsa

II Ventayo

II

II La Quebrada

II La Quebrada

I

II La Quebrada

III

II Çenboya

II La Quebrada

II Cuatonho

3 (2)

I

II La Quebrad

III

III

III

III Lile

III

III

I
II La Quebrada
Pontecui

Achindeta

Yqua

Ypiçe

Bono

Lama

Xeta

Talima

Petecui

Aça

Bola

Xita

Petecui

Aça

Yquinde

Bichicama

Cazcoli

Taqui

Piho

Petecui

Petecui

Xequino

Xequino

Yquinde

Condecoy

Condecoy

Petecui

Lama

Condecoy

Achindeta

Piho

Piho

Condecoy

Petecui

Quincha

Petecui

Pondo

Xita

Coao

Petecui

Petecui

Yquinde 
1.734 Oçacui

1.735 Oçe

1.736 Oçe

1.737 Oçecoy

1.738 Oçecui

1.739 Oçecui

1.740 Oçecui

1.741 Oçeçecui

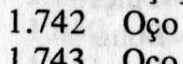

1.744 Oço

1.745 Oço

1.746 Oço

1.747 Oçombo

1.748 Oçoço

1.749 Ozcono

1.750 Ozcoy

1.751 Ozcoy

1.752 Ozcui

1.753 Pacui

1.754 Pacui

1.755 Pacha

1.756 Pagua

1.757 Pahande

1.758 Pajita

1.759 Pala

1.760 Pala

1.761 Pale

1.762 Pale

1.763 Pale

1.764 Palie

1.766 Pamoque

1.767 Pamua

1.768 Pana

1.769 Panança

1.770 Panche

1.771 Pandacui

1.772 Pandacui

1.773 Pandacui

1.774 Pandaxis

1.775 Pandica

1.776 Pandica

1.777 Pani

1.778 Pania
Diacuy

Bichicama

II Yoro

II Bocota

II Guahande

III Quiguata

II Guatonho

III

III

III

III

III Quiguata

II $\mathrm{Bex}$

III Lile

III Bitaco/Baça

III Lile

III Ventayo

III Bitaco/Andra

Yho

Yndegua

Chimba

Bola

Amalile

Achindeta

Pondo

Chiguatoco

Talima

Petecui

Chumba

Amalile

Bichicama

Yapo

Coao

Bichicama

Sali

Taqui

I Guahande
II Ventayo
III Lile
III Lile

III Lile

III

II Guatonho

II Amayme

I Guaales

I Guaales

III Quiguata

III

Dagua Benbacui

Petecui

II Bex

II Aguahax

II Cenboya

II La Quebrada

Petecui

Yapo

Yçando

Lama

Xequino

Yndegua
1.779 Panica

1.780 Panica

1.781 Panica

1.782 Panima

1.783 Panpacui

1.784 Pañudi

1.785 Paoa

1.786 Papa

1.787 Papa

1.788 Paquimbo

1.789 Paquimbo

1.790 Paquimbo

1.791 Paquimbo

1.792 Paquimbo

1.793 Paquimbo

1.794 Paquimbo

1.795 Paquimbo

1.796 Paquimbo

1.797 Paquinbo

1.798 Pata

1.799 Patac

1.800 Pate

1.801 Patre

1.802 Patre

1.803 Pauxa

1.804 Pauy

1.805 Paxando

1.806 Paxe

1.807 Paya

1.808 Paya

1.809 Payanda

1.810 Payande

1.811 Payando

1.812 Payando

1.813 Payando

1.814 Payogua

1.815 Payona

1.816 Pecna

1.817 Pecona

1.818 Peconta

1.819 Pecumba

1.820 Peghe

1.821 Pegua

.822 Pegua

1.823 Pela

1.824 Pelna

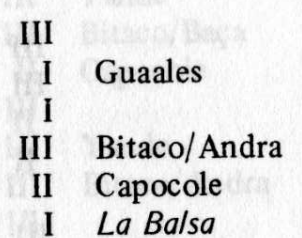

Petecui

(Alonso)

Xamundi

Ycobale

Cazcoli

Xita

Yndegua

Coao

Yçando

Pondo

Pondo

Petecui

Amalile

Amalile

Ycobale

Quincha

Bichicarna

Banbayaco

Petecui

Bonba

Aça

Xacoa

Ycobale

Achindeta

Bola

Taqui

Xacoa

Xacoa

Bola

Bonba

Achindeta

Achindeta

Achindeta

Bola

Bonba

Bonba

Achindeta

II Guatonho

III Bitaco/Andra

III Bitaco/Baça

III Ahoçetala

II Capocole

III Bitaco/Andra

II Bocota

III Quiguata

III
Yali

Ycobale

Chimba

Amalile

Petecui 